\title{
Phenomenology of Scalar Singlet Dark Matter in BSM Models
}

\author{
by \\ Robyn Campbell

\begin{abstract}
A thesis submitted to the
Faculty of Graduate and Postdoctoral Affairs

in partial fulfillment of the requirements for the degree of
\end{abstract}

Master of Science in Physics

Ottawa-Carleton Institute of Physics

Department of Physics

Carleton University

Ottawa, Canada

September 16, 2016

(C) Copyright

Robyn Campbell, 2016 


\section{Abstract}

Approximately $85 \%$ of the mass of the universe is classified as dark matter, a type of matter outside the framework of the Standard Model. This thesis studies the phenomenology of three models that incorporate dark matter. Firstly, a singlet scalar dark matter candidate is added to two previously established models: the Higgslike dilaton and the Georgi-Machacek models. The parameters of these models are constrained using experimental results such as dark matter direct detection measurements, Higgs signal strengths, Higgs invisible width and the measured relic abundance of dark matter in the universe. The notion of self-interacting dark matter, derived from measurements of galaxy cluster Abell 3827, is also studied for SSDM extensions of both the Standard Model and the Two Higgs Doublet model. The parameters of these models are in each case tightly constrained by experiment. 
for Maria 


\section{Acknowledgments}

I thank first and foremost my supervisor, Stephen Godfrey. Thank you for encouraging me to ask questions and for your patience in answering them.

I thank my collaborators: Alejandro de la Puente, Heather Logan, Alexandre Poulin, and Andrea Peterson. I appreciate the helpful discussions, quick answers, coding tips and life advice. I also thank my current and former lab mates for their guidance and also their enthusiasm for this field-it is certainly contagious!

I thank my undergraduate supervisors, Mohammed Ahmady and Ruben Sandapen for first introducing me to theoretical particle physics, and encouraging me to continue.

I thank my friends and church family, both in Ottawa and at home, for encouraging me to have a life outside Mathematica.

Finally, I thank my family. This thesis would not have been possible without your love and support, even when you had no idea what I was doing. 


\section{Preface}

Included in this thesis is a review of the author's work done as an M.Sc. student at Carleton University. All work contained within occurred only at Carleton University. Results of this work needed for analysis are included, but details identified as outside the scope of this thesis can be found in References [1-3].

Chapter 4 is entirely the work of the author, except for the Markov Chain Monte Carlo performed by Alejandro de la Puente. The fitting procedure was confirmed by comparison to a simple chi-squared fit to the ATLAS/CMS data.

The plots in Chapter 5 are accredited to Alexandre Poulin, as well are the details of the relic density scan.

All of the work in Chapter 6 was confirmed by the author's independent calculations, but Figure 6.4 and the ideas of thermal freeze-in and implementing the Two Higgs doublet model are accredited to the collaborators of Reference [1]. 


\section{Table of Contents}

Abstract $\quad$ ii

Acknowledgments $\quad$ iv

Preface $\quad$ V

Table of Contents $\quad$ vi

List of Tables $\quad$ ix

List of Figures $\quad$ x

1 Introduction 1

2 Beyond the Standard Model 3

2.1 Introduction to the Standard Model . . . . . . . . . . . . . . . . 3

2.1.1 Quantum Electrodynamics . . . . . . . . . . . . . . 4

2.1.2 Quantum Chromodynamics ............. 5

2.1.3 The Electroweak Theory and the Higgs Mechanism . . . . . . 6

2.2 Motivation for BSM Physics . . . . . . . . . . . . . . . . 9

2.2.1 The Hierarchy Problem . . . . . . . . . . . . . . . . . 9

2.2.2 Dark Matter . . . . . . . . . . . . . . . . . . . . 9

3 Experimental Constraints on Models $\quad 11$

3.1 Higgs Signal Strengths . . . . . . . . . . . . . . . . . . . . . . 11

3.2 Higgs Invisible Width . . . . . . . . . . . . . . . . . . . . 12

3.3 Dark Matter Relic Abundance . . . . . . . . . . . . . . . . . . 13

3.4 Dark Matter Direct Detection . . . . . . . . . . . . . . . . . 14 
4 Higgs-like Dilaton with Scalar Singlet DM 17

4.1 Background and Motivation . . . . . . . . . . . . . 17

4.2 Phenomenology . . . . . . . . . . . . . . . . . . . . . . 18

4.2.1 Higgs Signal Strengths . . . . . . . . . . . . . . . 18

4.2 .2 Higgs Invisible Width . . . . . . . . . . . . . . . . . 21

4.2.3 Dark Matter Relic Abundance . . . . . . . . . . . . . . . . . 22

4.2.4 Dark Matter Direct Detection . . . . . . . . . . . . . 26

4.3 Summary . . . . . . . . . . . . . . . . . . 28

5 Georgi-Machacek Model with Scalar DM 29

5.1 Background and Motivation . . . . . . . . . . . . . 29

5.2 Phenomenology . . . . . . . . . . . . . . . . . . . . . . 32

5.2.1 Theoretical Constraints . . . . . . . . . . . . . . 32

5.2 .2 Dark Matter Relic Density . . . . . . . . . . . . . . . 32

5.2 .3 Dark Matter Direct Detection . . . . . . . . . . . . . . . 37

5.2 .4 Dark Matter Invisible Width . . . . . . . . . . . . . . . . 38

5.2.5 Higgs Signal Strengths . . . . . . . . . . . . . . . . . . 39

5.3 Summary . . . . . . . . . . . . . . . . . . 40

6 Self-Interacting Dark Matter $\quad 42$

6.1 Background and Motivation . . . . . . . . . . . . . . . . . . 42

6.2 Minimal Singlet Extension of the Standard Model . . . . . . . . . . . 43

6.2.1 Heavy Dark Matter, $\mathrm{m}_{S} \gg \mathrm{m}_{H}$... . . . . . . . . . . 44

6.2.2 Dark matter near the Higgs resonance . . . . . . . . . . . . . 45

6.2.3 Low-mass dark matter, $\mathrm{m}_{S} \ll \mathrm{m}_{h}$. . . . . . . . . . . . . . . 47

6.2.4 Phenomenology . . . . . . . . . . . . . . . . . . . 47

6.3 Two Higgs Doublet Model with Singlet DM . . . . . . . . . . . . . . 50

6.3 .1 Type I $2 \mathrm{HDM} \ldots \ldots . \ldots \ldots$. . . . . . . . . . . 50

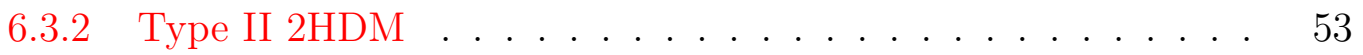

6.4 Summary .............................. 54

7 Summary $\quad 55$

$\begin{array}{ll}\text { List of References } & 57\end{array}$ 
Appendix A Feynman Rules

A.1 Higgs-like Dilaton with Scalar Singlet Dark Matter . . . . . . . . 61

A.2 Georgi-Machacek Model with Scalar DM . . . . . . . . . . . . . 62 


\section{List of Tables}

2.1 Known elementary interactions and their mediators [7]. . . . . . . . 3

4.1 Signal strengths for Higgs boson production channels according to ATLAS/CMS collab. [30], including predictions from the model in terms of $\epsilon$ and $c_{\gamma \gamma} \ldots \ldots \ldots \ldots \ldots \ldots \ldots$

4.2 Signal strengths for Higgs boson decay channels according to ATLAS/CMS collab. [30], including predictions from the model in terms

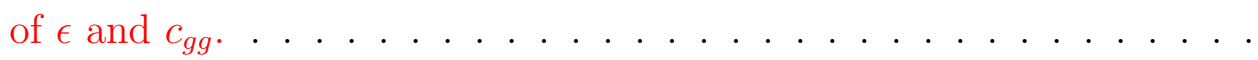




\section{List of Figures}

2.1 Elementary particles of the Standard Model . . . . . . . . . . . . . 4

2.2 An elementary vertex for QED . . . . . . . . . . . . 5

2.3 The composition of the universe, according to percentage by mass. . . 10

3.1 Feynman vertex for invisible Higgs decay . . . . . . . . . . . . . . . . 12

3.2 The Feynman diagram for DM direct detection through DM/nucleon scattering. . . . . . . . . . . . . . . . . 15

4.1 Parameter space of $c_{g g}$ and $c_{\gamma \gamma}$, where the allowed region is $\chi^{2}<5.9 . \quad 20$

4.2 The branching ratio for the dilaton decay to invisible particles, with $c_{g g}=0.67$ and $c_{\gamma \gamma}=-6.5$. The horizontal line is the ATLAS upper

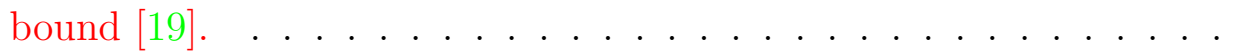

4.3 A contour plot of the $m_{S}$ and $c_{g g}$. The contours are the values for the branching ratio, with an experimental cutoff of 0.28 . The value of $c_{g g}$ is clearly not sensitive to the value of $m_{S}-$ no new information is gained concerning these parameters. . . . . . . . . . . .

4.4 The Feynman diagrams for the processes $\mathrm{SS} \rightarrow \mathrm{xx}$, where $\mathrm{x}$ is a $\mathrm{SM}$

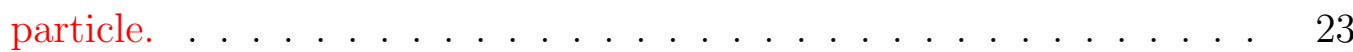

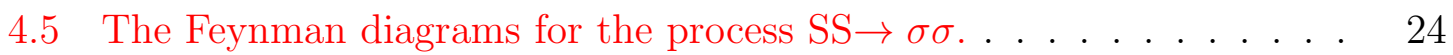

4.6 The DM relic abundance as a function of the DM mass. The $\lambda=3$ case corresponds to the black line, and the $\lambda=4 \pi$ case corresponds to the lower grey line. The horizontal grey line is the experimental value $[21] \ldots \ldots \ldots \ldots \ldots \ldots$

4.7 The DM direct detection cross section as a function of the DM mass. The cases for $\lambda=3$ and $\lambda=4 \pi$ are shown in black and dark grey, respectively. The experimental limits are, starting from bottom: DEAP projection (black), LUX (grey), and XENON (light grey). . . . . . . 27

5.1 Feynman diagram for $S S \rightarrow A+B$ where A, B are SM particles . . . 32

5.2 Feynman diagram for $S S \rightarrow H_{3} V^{(*)} \ldots \ldots \ldots 33$ 
5.3 Feynman diagrams for $S S \rightarrow h_{1} h_{2} \ldots \ldots \ldots \ldots \ldots \ldots$

5.4 The average and standard deviation of critical parameters . . . . . 35

5.5 Feynman diagram for direct detection . . . . . . . . . . . 37

5.6 Direct detection cross section as a function of $m_{S}$. The left plot is a zoom of the low- $m_{S}$ region while the right plot shows the full $m_{S}$ range scanned. Experimental bounds include LUX (magenta), XENON10 (purple), XENON1T (brown), DEAP3600 (green), and the neutrino

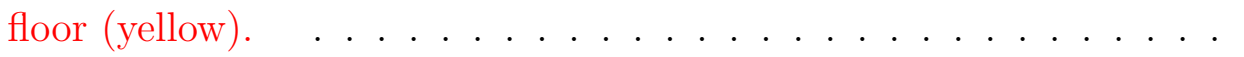

5.7 Constraint from invisible decays of the Higgs for a dark matter mass range between $0 \mathrm{GeV}$ and $125 / 2 \mathrm{GeV}$. The blue points are allowed while the red points are excluded by either direct detection constraints, $b \rightarrow s \gamma$ constraints, constraints on the invisible decays of the Higgs, dwarf spheroidal galaxy constraints, or mass constraints on the triplet and fiveplet. Anything above the blue line is excluded. . . . . . . .

5.8 Parameter space of $\kappa_{f}$ and $\kappa_{V}$. The blue points are allowed by all previously imposed constraints. . . . . . . . . . . . .

6.1 Abell 3827 HST image from Reference [42], showing the F160W (red), F160W (green) and F336W (blue) optical bands on a logarithmic colour scale. Labels N1 through N4 are the four bright central galaxies, while N5 is a star. . . . . . . . . . . . . . . . . . . 4 43

6.2 Feynman diagrams for the interaction $S S \rightarrow S S \ldots \ldots \ldots \ldots$

6.3 Feynman diagrams for the annihilation of DM into SM particles in the real singlet scalar extension of the SM. . . . . . . . . . . .

6.4 The allowed region for $m_{S}$ and $\left|\lambda_{\text {eff }}\right|$ to obtain the desired range in Equation 6.8. This includes the constraints from perturbative unitarity and thresholds for DM annihilation to electrons, muons, and neutral

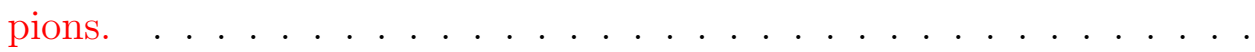




\section{Chapter 1}

\section{Introduction}

During the press release announcing the detection of gravitational waves, LIGO Scientific Collaboration (LSC) spokesperson Gabriela Gonzalez called the discovery "the beginning of a new era" in gravitational wave astronomy [4]. This is not the only area of fundamental physics that has experienced major discoveries over the past few years. The discovery of the Higgs Boson in 2012 confirmed the 50-year-old prediction of its existence and resulted in a Nobel prize [5]. In late 2015, the Large Hadron Collider (LHC) began operation at $13 \mathrm{TeV}$, and has already produced a signal exciting theorists, namely the hints of a $750 \mathrm{GeV}$ diphoton excess [6].

Particle identification and interaction are neatly intertwined into a framework called the Standard Model (SM). Current experiment, fuelled by inconsistencies and gaps in this framework, searches for physics beyond the Standard Model (BSM). For example, there exists a question of scale in the SM: why do the masses have the values they do and why are there no intermediate natural scales between the electroweak and Planck scales? One of the most intriguing BSM probes lies in the fields of dark matter and dark energy - humbling detections indicating a lack of knowledge of $95 \%$ of the mass of the universe.

Theorists all over the world seek to remedy the ailments of the Standard Model. Some decide to start over and build a new model from scratch, such as string theory. Many take the more conservative approach and build on the existing foundation by introducing new particles and fields, where examples include supersymmetry, extending the Higgs sector, and adding extra dimensions. Of the two approaches this thesis will study the latter, considering select SM extensions that are expanded to include a singlet scalar dark matter (SSDM) candidate.

This thesis is structured as follows: 
Chapter 2 is an overview of the Standard Model: its particles, forces, and mechanisms. It also introduces motivation for BSM physics, namely the hierarchy problem and dark matter. Chapter 3 examines the experimental measurements that will be used to constrain the proposed models.

The next three chapters examine three different models that incorporate dark matter candidates.

Chapter 4 considers a model with a Higgs-like dilaton and singlet scalar dark matter. Chapter 5 couples the well-established Georgi-Machacek model to an additional singlet serving as a dark matter candidate. In Chapter 6 self-interacting dark matter is examined, which stems from a dark matter halo offset in galaxy cluster Abell 3827. Results are summarized in Chapter 7. 


\section{Chapter 2}

\section{Beyond the Standard Model}

\subsection{Introduction to the Standard Model}

The Standard Model (SM) of particle physics provides a framework for interactions of elementary particles. There are four known fundamental interactions: electromagnetic, strong, weak, and gravitational. Properties of the four forces, including relative strength and spin, are listed in Table 2.1.

\begin{tabular}{|c|c|c|c|}
\hline Interaction & Mediator & Spin & Relative Strength \\
\hline Gravitational & Graviton & 2 & $10^{-42}$ \\
\hline Weak & $\mathrm{W}^{ \pm}, \mathrm{Z}$ & 1 & $10^{-13}$ \\
\hline Electromagnetic & Photon & 1 & $10^{-2}$ \\
\hline Strong & Gluon & 1 & 10 \\
\hline
\end{tabular}

Table 2.1: Known elementary interactions and their mediators [7].

The Standard Model unifies the strong, weak, and electromagnetic interactions. Individual gravitons have not yet been discovered and there is yet to be a consistent theory of quantum gravity.

The known elementary particles are listed in Figure 2.1. The Dirac equation also predicts for each particle an antiparticle, or one of the same mass and spin but opposite charge. There are three leptons and three lepton neutrinos, of which only the electron is stable. It is believed that charge is preserved in all interactions, as is lepton number apart from neutrino oscillations. 

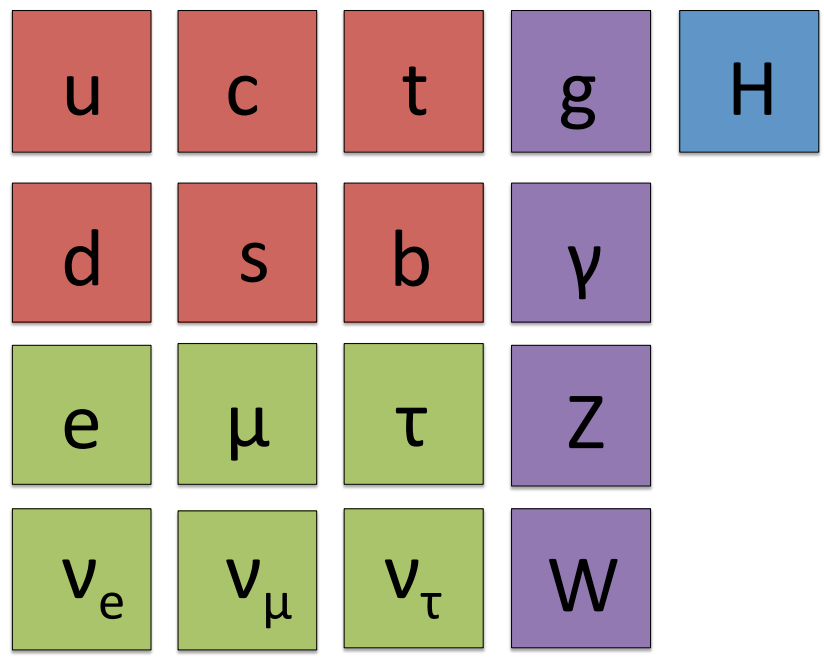

Figure 2.1: Elementary particles of the Standard Model

Quarks are found only in bound states called hadrons. Hadrons can be classified further as either mesons (a quark and an antiquark) or baryons (three quarks). There is also evidence of four-quark systems, and recently bound states of five quarks, aptly named pentaquarks, may have been discovered [8]. All mesons are unstable, and the only stable baryon is the proton.

There are also four gauge bosons: gluons, which carry the strong interaction, photons, which carry the electromagnetic interaction, and the $\mathrm{W}$ and $\mathrm{Z}$ bosons, which together carry the weak interaction. Finally, the SM includes the Higgs boson, which serves to give the other particles mass. Details of these mechanisms can be found in the following sections.

\subsubsection{Quantum Electrodynamics}

Quantum electrodynamics (QED) is an extremely successful dynamical theory, mediating the interactions between photons and matter. Every interaction can be reduced to an elementary vertex of the form $\gamma \rightarrow q q$, where $q$ is any charged particle. The interaction governed by QED of a photon with an electron is shown in Figure 2.2. The QED Lagrangian is

$$
\mathcal{L}_{Q E D}=\bar{\psi}(i \not D-m) \psi-\frac{1}{4}\left(F_{\mu \nu}\right)^{2}
$$




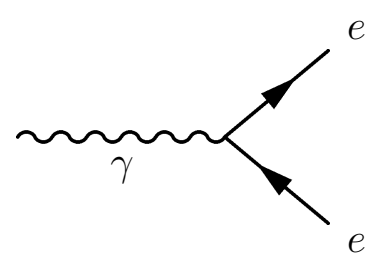

Figure 2.2: An elementary vertex for QED

where $\psi$ is a field of spin-1/2 particles of mass $m$, and $D_{\mu}$ is the gauge covariant derivative

$$
D_{\mu}=\partial_{\mu}+i e A_{\mu}(x)
$$

Here, $A_{\mu}$ is the electromagnetic vector potential, such that the electromagnetic field tensor $F_{\mu \nu}$ is defined as [9]

$$
F_{\mu \nu}=\partial_{\mu} A_{\nu}-\partial_{\nu} A_{\mu}
$$

The Lagrangian is invariant under the gauge transformations

$$
\begin{array}{r}
A_{\mu} \rightarrow A_{\mu}+\partial_{\mu} \lambda \\
\psi \rightarrow e^{-i e \lambda} \psi .
\end{array}
$$

The electric charge $e$ is typically written in terms of the fine structure constant, $\alpha$ :

$$
\alpha=\frac{e^{2}}{4 \pi \hbar c} \approx \frac{1}{137}
$$

QED is valid for all electromagnetic phenomena observed in nature, such as Compton scattering, Bhabha scattering, and electron-positron annihilation.

\subsubsection{Quantum Chromodynamics}

Quantum chromodynamics (QCD) describes the interactions of coloured particles: quarks and gluons. Quarks can either be red (r), green (g), or blue (b), and antiquarks can be the corresponding anti-colours. Interactions proceed via the exchange of gluons, and is dependent on the strength of the strong coupling constant $\alpha_{s}$.

The QCD portion of the Standard Model is as follows: 


$$
\mathcal{L}_{Q C D}=-\frac{1}{4} F_{\mu \nu}^{a} F^{\mu \nu a}+i \sum_{q} \bar{\phi}_{q}^{i} \gamma^{\mu}\left(D_{\mu}\right)_{i j} \phi_{q}^{j}-\sum_{q} m_{q} \bar{\phi}_{q}^{i} \phi_{q i}
$$

where

$$
F_{\mu \nu}^{a}=\partial_{\mu} A_{\nu}^{a}-\partial_{\nu} A_{\mu}^{a}-g_{s} f_{a b c} A_{\mu}^{b} A_{\nu}^{c}
$$

and

$$
\left(D_{\mu}\right)_{i j}=\delta_{i j} \partial_{\mu}+i g_{s} \sum_{a} \frac{\lambda_{i j}^{a}}{2} A_{\mu}^{a} .
$$

Here, $\phi_{q}^{i}$ are the quark fields with colour $i$ and flavour $q, A_{\mu}^{a}$ are the gluon fields, $g_{S}$ is the strong coupling strength (ie $g_{S}=\sqrt{4 \pi \alpha_{S}}$ ), $f_{a b c}$ are the $\mathrm{SU}(3)$ structure constants, and $\lambda_{i j}^{a}$ are the Gell-Mann matrices [9].

\subsubsection{The Electroweak Theory and the Higgs Mechanism}

This section follows the combined notation of [10] and [11]. The Higgs Mechanism is introduced as the means to give fermions and gauge bosons mass. Consider the Glashow-Weinberg-Salam theory of electroweak interactions, containing three $\mathrm{SU}(2)_{L}$ gauge bosons $W_{\mu}^{i}(i=1,2,3)$, and one $\mathrm{U}(1)_{Y}$ gauge boson $B_{\mu}$. The Lagrangian is

$$
\mathcal{L}=\left(D^{\mu} \Phi\right)^{\dagger}\left(D_{\mu} \Phi\right)-V(\Phi)-\frac{1}{4} W_{\mu \nu}^{i} W^{\mu \nu i}-\frac{1}{4} B_{\mu \nu} B^{\mu \nu}
$$

where $D_{\mu}$ is the covariant derivative associated to $\mathrm{SU}(2)_{L} \times \mathrm{U}(1)_{Y}$

$$
D_{\mu}=\partial_{\mu}+i g^{\prime} \frac{Y}{2} B_{\mu}+i g \tau^{i} \cdot \vec{W}_{\mu}^{i}
$$

and $\Phi$ is the complex scalar doublet

$$
\Phi=\left(\begin{array}{c}
\Phi_{+} \\
\Phi_{0}
\end{array}\right) .
$$

The simplest potential that is renormalizable and $\mathrm{SU}(2)_{L}$ invariant is

$$
V=-\mu^{2}\left|\Phi^{\dagger} \Phi\right|+\lambda\left(\left|\Phi^{\dagger} \Phi\right|\right)^{2} .
$$

A ground state at the minimum of $\mathrm{V}$ is chosen, such that 


$$
\left|\Phi_{0}\right|=\frac{\mu}{\sqrt{2 \lambda}}, \quad\left|\Phi_{+}\right|=0 .
$$

The unitary gauge is now used to avoid explicit Goldstone bosons, and these degrees of freedom are transformed into longitudinal components of massive gauge bosons. The symmetry is broken spontaneously by introducing the vacuum expectation value (vev):

$$
\langle\Phi\rangle=\sqrt{\frac{\mu^{2}}{2 \lambda}} \equiv \frac{v}{\sqrt{2}}
$$

It is then convenient to rewrite $\Phi$ as

$$
\Phi=\frac{1}{\sqrt{2}}\left(\begin{array}{c}
0 \\
v+h
\end{array}\right) .
$$

The Lagrangian in Equation 2.9 is invariant under the $\mathrm{SU}(2)$ transformation $\Phi \rightarrow$ $-\Phi$, however the Lagrangian is not invariant under the transformation $\mathrm{h} \rightarrow-\mathrm{h}$. Therefore, the electroweak symmetry is spontaneously broken. After substitution of 2.14 into the Lagrangian, which is then expanded up to second order terms, four physical fields emerge: two charged fields, $\mathrm{W}^{ \pm}$, and two neutral fields, $\mathrm{Z}$ and $\gamma$ :

$$
\begin{aligned}
W_{\mu}^{ \pm} & =\frac{1}{\sqrt{2}}\left(W_{\mu}^{1} \mp i W_{\mu}^{2}\right) \\
Z^{\mu} & =\frac{-g^{\prime} B_{\mu}+g W_{\mu}^{3}}{\sqrt{g^{2}+g^{\prime 2}}} \\
A^{\mu} & =\frac{g B_{\mu}+g^{\prime} W_{\mu}^{3}}{\sqrt{g^{2}+g^{\prime 2}}} .
\end{aligned}
$$

The Lagrangian can be rewritten in terms of these new fields

$$
\mathcal{L}=\left(D^{\mu} \phi\right)^{\dagger}\left(D_{\mu} \phi\right)-\frac{1}{8} v^{2}\left(g^{2}\left(W^{+}\right)^{2}+g^{2}\left(W^{-}\right)^{2}+\left(g^{2}+g^{\prime 2}\right) Z_{\mu}^{2}+0 \cdot A_{\mu}^{2}\right)
$$

from which the masses of the gauge bosons can be extracted. They are as follows:

$$
m_{W}^{2}=\frac{1}{4} g^{2} v^{2}
$$




$$
\begin{gathered}
m_{Z}^{2}=\frac{1}{4}\left(g^{2}+g^{\prime 2}\right) v^{2} \\
m_{A}=0 .
\end{gathered}
$$

This also defines the weak mixing angle $\theta_{W}$ by way of the coupling constants and the electromagnetic strength e:

$$
\begin{aligned}
& e=g \sin \theta_{W} \\
& e=g^{\prime} \cos \theta_{W} .
\end{aligned}
$$

As an example, consider the fermion masses. The Yukawa Lagrangian is as follows:

$$
\mathcal{L}_{\text {Yuk }}=\Gamma_{m n}^{u} \bar{q}_{m, L} \tilde{\phi} u_{n, R}+\Gamma_{m n}^{d} \bar{q}_{m, L} \phi d_{n, R}+\Gamma_{m n}^{e} \bar{l}_{m, L} \phi e_{n, R}+\text { h.c. }
$$

where the matrices $\Gamma_{m n}$ contain the Yukawa couplings. Since the mass terms must be without hypercharge, there must be two representations of Higgs fields, $\phi$ and $\tilde{\phi}$, in order to give mass to the down type and up type fermions separately. In short, the Yukawa Lagrangian can be rewritten for the first generation:

$$
\mathcal{L}_{\text {Yuk }}=f_{e} \bar{l}_{L} \phi e_{R}+f_{u} \bar{q}_{L} \tilde{\phi} u_{R}+f_{d} \bar{q}_{L} \phi d_{R}+\text { h.c. }
$$

which is, as before, in the unitary gauge. Substituting the fields into the Lagrangian gives the convenient form

$$
\mathcal{L}_{Y u k}=\frac{f_{e} v}{\sqrt{2}}\left(\bar{e}_{L} e_{R}+\bar{e}_{R} e_{L}\right)+\frac{f_{u} v}{\sqrt{2}}\left(\bar{u}_{L} u_{R}+\bar{u}_{R} u_{L}\right)+\frac{f_{d} v}{\sqrt{2}}\left(\bar{d}_{L} d_{R}+\bar{d}_{R} d_{L}\right) .
$$

This gives an expression for the mass terms:

$$
m_{i}=-\frac{f_{i} v}{\sqrt{2}}, \quad i=e, u, d .
$$




\subsection{Motivation for BSM Physics}

\subsubsection{The Hierarchy Problem}

The Higgs mechanism is the driving force behind electroweak symmetry breaking (EWSB), but theorists hesitate to accept it as a complete theory. It does not explain the physical value of the vev $(\mathrm{v}=246 \mathrm{GeV})$, or why fermions have the masses they do. The most serious problem, however, is that first order loop corrections to the Higgs mass are quadratically divergent [12]. If we define $m_{H}^{2}=m_{H_{0}}^{2}+\delta m_{H}^{2}$, the loop correction at the one-loop level reads

$$
\delta m_{H}^{2}=\frac{G_{F} \Lambda^{2}}{4 \sqrt{2} \pi^{2}}\left(6 m_{W}^{2}+3 m_{Z}^{2}+m_{H}^{2}-12 m_{t}^{2}\right)+\ldots
$$

where $\Lambda^{2}$ is the ultraviolet (UV) scale boundary of the SM. The only natural scale found in the SM is the Planck scale, or $\Lambda=M_{\text {Planck }} \approx 10^{19} \mathrm{GeV}$. In order to produce results comparable to the order of the Higgs mass, fine-tuned counterterms must be added to cancel the divergences.

Alternatively, we can assume that the correction is on the order of the observed $125 \mathrm{GeV}$ Higgs boson, meaning the scale $\Lambda$ is less than the Planck scale. This implies that $\Lambda$ is on the order of $1 \mathrm{TeV}$, so one would expect to see new physics at about this scale. The little hierarchy problem arises due to the fact that much of the new physics one expects to see is ruled out by experimental searches [13].

\subsubsection{Dark Matter}

There is overwhelming evidence that the matter observed in the Standard Model makes up only approximately $4 \%$ of the mass of the universe (see Figure 2.3). The remainder is dark energy $(\sim 73 \%)$ and dark matter $(\sim 23 \%)$ [14]. Dark energy is a repulsive force aiding in the acceleration of the expansion of the universe. A number of solutions have been proposed to this mystery, such as alterations to the cosmological constant and additional scalar fields. This thesis does not concern itself with dark energy, but instead focuses on dark matter.

The initial evidence for dark matter is found in a paper written by Fritz Zwicky in 1937 [15]. The argument states that estimates of nebulae masses based on their luminosities and rotation curves are inconsistent. Measurements of the Coma cluster 


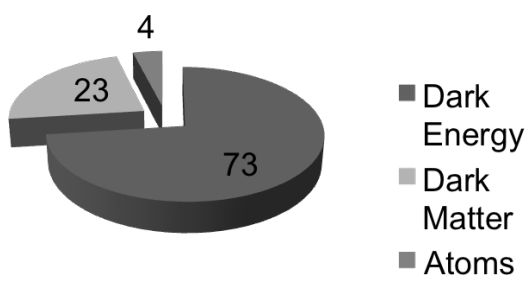

Figure 2.3: The composition of the universe, according to percentage by mass.

show that its galaxies cannot be gravitationally bound to the cluster by the force of the visible matter alone. Given its radical conclusion, Zwicky's paper only received about 10 citations in the first forty years after its publication [16].

The notion of dark matter was revived with Vera Rubin in the 1970's. She found that the rotational speed of a galaxy remains constant past the visible edge of the galaxy, where classical physics expects it to decrease. This indicates that most of the mass in a galaxy is contained in a spherical "dark halo" around the edge of the galaxy [17]. There exists additional evidence for the existence of dark matter, for example, gravitational lensing, the Cosmic Microwave background, and the large-scale structure of the universe [14].

Numerous DM particle candidates have been proposed. Most are Standard Model additions, coming into effect at the electroweak scale. Some hinge off other models, such as supersymmetry or string theory. However, as of yet, a dark matter particle has not been observed by any experiment- the best one can do is interpret what its absence reveals about its nature.

It is known that a dark matter candidate must be weakly interacting but still able to exert a gravitational pull. It does not reflect, emit or absorb light. The particle has a known energy density and galactic velocity, and it freezes out its relic abundance. There are very few limits on the mass of a dark matter particle, but a phenomena known as the "WIMP miracle" dictates that the self annihilation cross section required to obtain the correct abundance of dark matter today suggests a particle in the GeV mass range, which can be probed by colliders [14]. 


\section{Chapter 3}

\section{Experimental Constraints on Models}

To explore possibilities beyond the Standard Model, new models are proposed to remedy one or more of the previously posed issues with the current framework. However, it is preferred that these extensions be testable with existing experimental data, or else have the potential to guide future experiments. This chapter introduces the phenomenological tools used in this thesis, used to constrain parameters of the models in the subsequent sections.

\subsection{Higgs Signal Strengths}

The signal strengths are defined as:

$$
\mu_{i}=\frac{\sigma_{i}}{\left(\sigma_{i}\right)_{S M}} \quad \text { and } \quad \mu^{f}=\frac{B R^{f}}{\left(B R^{f}\right)_{S M}}
$$

where the $\sigma_{i}$ are Higgs boson production cross sections for initial states $\mathrm{i}$ and the $\mathrm{BR}^{f}$ are decay branching ratios for final states f. The $S M$ subscripts indicate the SM predictions for the cross sections and branching ratios, such that the expected signal strength values are $\mu_{i}=\mu^{f}=1$.

The ATLAS/CMS collaborations provide numerical results for the values of $\mu_{i}$ and $\mu^{f}$ [18]. They present a combined fit to both the $\sqrt{s}=7$ and $\sqrt{s}=8$ data, assuming that the signal strength is the same between the two energies.

The main production modes studied are gluon fusion $(\mathrm{ggF})$, vector boson fusion $(\mathrm{VBF})$, and associated production with vector bosons $(\mathrm{VH})$ or a pair of top quarks $(\mathrm{ttH})$. The decay modes considered are decays to $\mathrm{Z}$ bosons, $\mathrm{W}$ bosons, photons, $\tau$ leptons, bottom quarks, and muons. In general, we assume the measured particle 
mass of $125.09 \mathrm{GeV}$ has the properties of the SM Higgs. A comparison can be done between the experimental results and the values predicted by a given model, often invoking a $\chi^{2}$ fit or other fitting procedure. Since the signal strength results are applied slightly differently between models in this thesis, procedural details will be included in their respective chapters.

\subsection{Higgs Invisible Width}

The process $h \rightarrow S S$ (Figure 3.1) where $\mathrm{S}$ is the dark matter candidate provides a constraint on the dark matter in the lower mass range $m_{S}<m_{h} / 2$. This process is invisible to the detector apart from large missing energy. The invisible width of a

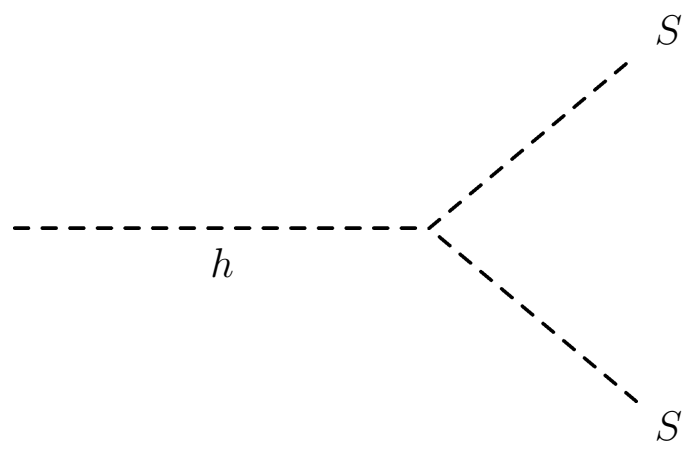

Figure 3.1: Feynman vertex for invisible Higgs decay

Higgs boson, or the probability of a Higgs decaying invisibly to two DM candidates, is in many cases a simple calculation given the Higgs-DM coupling. It can be written in the generic form

$$
\Gamma(h \rightarrow S S)=\frac{g_{h S S}^{2}}{32 m_{h}^{2} \pi} \sqrt{m_{h}^{2}-4 m_{S}^{2}}
$$

where $i g_{h S S}$ is the Feynman coupling for the hSS vertex. Experimentally, we are interested in the branching ratio, which also depends on the total width of the Higgs boson to standard model particles $\Gamma(h \rightarrow S M)$ :

$$
B R(h \rightarrow \text { invis. })=\frac{\Gamma(h \rightarrow S S)}{\Gamma(h \rightarrow S M)+\Gamma(h \rightarrow S S)} .
$$

The ATLAS experiment bounds the invisible Higgs branching ratio from above. 
This constraint comes from direct searches for Higgs decays in dilepton or dijet final states with missing transverse momentum at $\sqrt{s}=8 \mathrm{TeV}$. There are three mechanisms considered, combined statistically to give the final result:

- the Higgs boson is produced by itself by VBF, and decays invisibly. The signature of this process is two jets with a large separation in pseudorapidity, and large missing transverse momentum.

- the Higgs boson is produced with a Z boson, which decays to leptons while the Higgs decays invisibly. The signature of this process is opposite signed, same flavoured leptons and large missing transverse momentum.

- the Higgs boson is produced with a vector boson ( $\mathrm{Z}$ or $\mathrm{W})$, which decays to jets while the Higgs decays invisibly. The signature of this process is two jets where the invariant mass of the jets is consistent with the boson mass, and large transverse momentum

The model independent observed upper limit at the $95 \% \mathrm{CL}$ is $B R(h \rightarrow$ invis. $)<0.28$ [19].

\subsection{Dark Matter Relic Abundance}

In the early universe, there was an abundance of high mass, high energy particles created and annihilated in thermal equilibrium. However, due to the expansion and cooling of the universe, the equilibrium was lost and the number density became constant. This is know as thermal "freeze out" and occurs when the Hubble expansion rate is equal to the reaction rate, which can be found using the Boltzmann equation for the comoving particle density [20]

$$
\frac{d n_{S}}{d t}+3 H n_{S}=-\langle\sigma v\rangle\left(n_{S}^{2}-\left(n_{S}^{e q}\right)^{2}\right),
$$

where $H$ is the Hubble parameter, $\sigma$ is the DM annihilation cross section, $v$ is the

relative velocity, $n_{S}$ is the number density of $S$, and $n_{S}^{e q}$ is the equilibrium number density of $S$. The bracket notation $\langle\sigma v\rangle$ indicates the thermal average of the enclosed cross section. 
The freezeout temperature, $T_{F O}$, can be found by analytically solving the transcendental equation

$$
\left.\frac{m_{S}}{T_{F O}} \approx \ln \left(\frac{0.038 g_{S} m_{S} M_{p l}}{g^{\frac{1}{2}}\left(\frac{m_{s}}{T_{F O}}\right)^{\frac{1}{2}}}\langle\sigma v\rangle\right)\right|_{T=T_{F O}}
$$

where the thermalized cross section is given by

$$
\langle\sigma v\rangle=\frac{1}{8 m_{S}^{4} T K_{2}^{2}\left(\frac{m_{S}}{T}\right)} \int_{4 m_{s}^{2}}^{\infty} d s \sigma(s)\left(s-4 m_{S}^{2}\right) s^{\frac{1}{2}} K_{1}\left(\frac{s^{\frac{1}{2}}}{T}\right) .
$$

Here, $K_{1}(z)$ and $K_{2}(z)$ are modified Bessel functions of the second kind, and $M_{p l}$ is the Planck mass.

The value of $x=m_{S} / T_{F O}$ is typically in the range $\{4,40\}$. The thermalized cross section can then be evaluated at the freeze out temperature and inserted into the expression for relic abundance, which gives

$$
\Omega h^{2}=1.65 \times\left. 10^{-10}\left(\frac{1}{\langle\sigma v\rangle} \log \left(\frac{0.038 g_{S} m_{S} M_{p l}}{\sqrt{g}}\langle\sigma v\rangle\right)\right)\right|_{T=T_{F O}}
$$

where $g_{S}$ is the number of degrees of freedom for the dark matter candidate (equals 1 for SSDM) and $g=86$ is the number of relativistic degrees of freedom at the freezeout temperature. Here, $\Omega$ is the critical energy density of the universe and $h$ is the Hubble parameter.

The Planck Collaboration measured the value to be $\Omega h^{2}=0.1199 \pm 0.0027$ by mapping anisotropies of the cosmic microwave background (CMB) [21]. The predicted value of the relic density for a given model must be less or equal to the experimental value, as a deficiency can potentially be accounted for by adding additional DM candidates.

\subsection{Dark Matter Direct Detection}

If a dark matter candidate comes into close enough proximity to a nucleon, a momentum transfer may occur by exchange of a Higgs boson (see Figure 3.2). The nuclear recoil, however, is small: a WIMP with a mass of $10-1000 \mathrm{GeV} / \mathrm{c}^{2}$ generates a recoil in the range of $1-100 \mathrm{keV}$ [22]. 


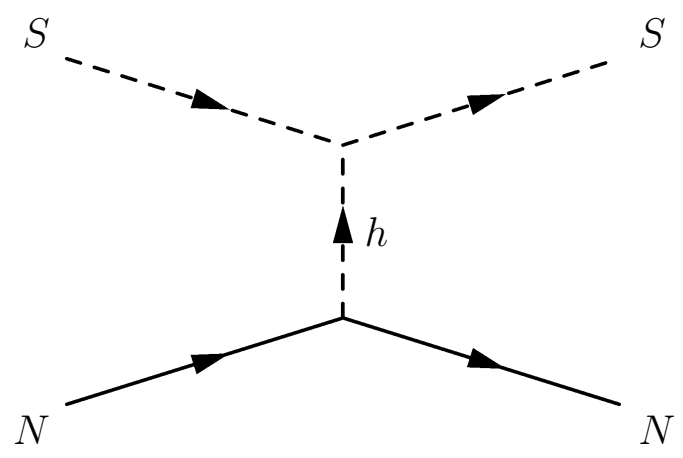

Figure 3.2: The Feynman diagram for DM direct detection through DM/nucleon scattering.

The general form of the direct detection cross section is

$$
\sigma=\frac{f_{N}^{2} g_{h S S}^{2} m_{N}^{4}}{4 \pi\left(m_{N}+m_{S}\right)^{2} m_{h}^{4} v^{2}}
$$

where $i f_{N}$ is the Feynman coupling for the Higgs-nucleon vertex. The experimental values used for comparison are from the XENON and LUX experiments [23,24]. Both are underground liquid xenon detectors, generating and recording scintillation light from reactions. A time lapse occurs between the initial reaction and a secondary reaction generated by free electrons, making it possible to localize events in three coordinates. The detectors are placed deep underground to minimize background from cosmic rays $[25,26]$. Projected limits are also included from the DEAP 3600 experiment [27].

In order to apply direct detection searches, a number of assumptions must be made:

- the halo of DM particles is smooth, and can be described by Maxwell velocity distributions

- the galactic disk rotates, therefore there exists an annual modulation due to the Earth's orbital motion to compensate for the so-called "WIMP wind"

- the nucleus is a hard sphere, described by Helm form factor

- the scattering is elastic 
It is not the recoil energy that is measured directly, but the recoil energy distribution, creating a DM density profile [28]. 


\section{Chapter 4}

\section{Higgs-like Dilaton with Scalar Singlet DM}

\subsection{Background and Motivation}

Classically, the Standard Model is scale invariant apart from the Higgs mass, which can be generated naturally at the electroweak scale by a soft breaking of the scale invariance. However, breaking non-linear scale invariance spontaneously implies the existence of a Goldstone boson, the dilaton. If the dimensionful operators are near marginal at the breaking scale, the dilaton can be naturally light.

The model studied in this chapter considers a Higgs-like dilaton, that is, a dilaton with properties of the $125 \mathrm{GeV}$ Higgs boson [29]. It is further extended by a singlet scalar dark matter candidate. We take the simplest addition, a real scalar field, which only interacts with standard model particles through the dilaton portal.

In this model, to find the couplings of the dilaton to the SM fields and the scalar dark matter candidate we follow the procedure of non-linearly realized scale invariance. The potential is [2]

$$
\begin{gathered}
V(\bar{\chi}, S) \approx \frac{1}{2} m_{\sigma}^{2} \bar{\chi}^{2}-\frac{\lambda}{3 !} \frac{m_{\sigma}^{2}}{f} \bar{\chi}^{3}+\frac{\bar{\chi}}{f} \sum_{i}\left(1+\gamma_{\psi_{i}}\right) m_{\psi_{i}} \bar{\psi}_{i} \psi_{i}+\left(\frac{2 \bar{\chi}}{f}+\frac{\bar{\chi}^{2}}{f^{2}}\right) \\
\times\left[m_{W}^{2} W^{+\mu} W_{\mu}^{-}+\frac{1}{2} m_{Z}^{2} Z^{\mu} Z_{\mu}\right]+\frac{\alpha_{\mathrm{EM}}}{8 \pi f} c_{\gamma \gamma} \sigma F_{\mu \nu} F^{\mu \nu}+\frac{\alpha_{\mathrm{S}}}{8 \pi f} c_{g g} \sigma G_{\mu \nu} G^{\mu \nu} \\
+\frac{1}{2} \bar{m}_{S}^{2} S^{2}+\lambda_{S} S^{4}
\end{gathered}
$$

where the dilaton $(\sigma)$ is parametrized with the non-linear realization as $\chi=f e^{\sigma / f}$, and dilaton is expanded about its vev as $\bar{\chi}=\chi-f$, while $\bar{m}_{S}^{2}=m_{S}^{2} e^{2 \sigma / f}$. The 
exponentials are expanded to leading orders to obtain the following parametrization for the potential:

$$
\begin{gathered}
V(\sigma, S) \approx \frac{1}{2} m_{\sigma}^{2} \sigma^{2}+\epsilon \frac{m_{\sigma}^{2}}{v}\left(\frac{1}{2}-\frac{1}{6} \lambda\right) \sigma^{3}+\frac{\sigma}{f} \sum_{i}\left(1+\gamma_{\psi_{i}}\right) m_{\psi_{i}} \bar{\psi}_{i} \psi_{i}+\left(\frac{2 \sigma}{f}+\frac{\sigma^{2}}{f^{2}}\right) \\
{\left[m_{W}^{2} W^{+\mu} W_{\mu}^{-}+\frac{1}{2} m_{Z}^{2} Z^{\mu} Z_{\mu}\right]+\frac{\alpha_{\mathrm{EM}}}{8 \pi f} c_{\gamma \gamma} \sigma F_{\mu \nu} F^{\mu \nu}+\frac{\alpha_{\mathrm{S}}}{8 \pi f} c_{g g} \sigma G_{\mu \nu} G^{\mu \nu}} \\
+\frac{1}{2} m_{S}^{2}\left(1+2 \epsilon \frac{\sigma}{v}+2 \epsilon^{2} \frac{\sigma^{2}}{v^{2}}\right) S^{2}+\lambda_{S} S^{4}
\end{gathered}
$$

where $f$ denotes the scale where the conformal symmetry is spontaneously broken, $v$, the EWSB scale and $\epsilon=v / f$. The parameter $\lambda$ is related to the trilinear dilaton coupling. A $\mathrm{Z}_{2}$ symmetry is imposed under which $\mathrm{S}$ is odd while all other fields are even, ensuring that the dark matter candidate is stable. The parameter $\lambda_{S}$ is a free parameter unable to be constrained by experiment at this time.

To summarize, this theory depends on six independent parameters: $m_{S}, \lambda, \lambda_{S}, \epsilon$, $c_{g g}$ and $c_{\gamma \gamma}$ where

$$
\begin{aligned}
c_{g g} & =\left(b_{I R}^{3}-b_{U V}^{3}\right) \\
c_{\gamma \gamma} & =\left(b_{I R}^{E M}-b_{U V}^{E M}\right) .
\end{aligned}
$$

Here, $b_{U V}$ and $b_{I R}$ represent the ultraviolet and infrared contributions to the running of the EM and strong gauge couplings respectively at a scale above conformal symmetry breaking. At high energies, the underlying theory is not considered and the SM loop contribution to $c_{g g}$ and $c_{\gamma \gamma}$ are absorbed in the definition.

\subsection{Phenomenology}

\subsubsection{Higgs Signal Strengths}

The latest data at $8 \mathrm{TeV}$ from the ATLAS and CMS collaborations shown in Tables 4.1 and 4.2 is used to constrain the dilaton with measured Higgs couplings. 


\begin{tabular}{lccccc}
\hline & $\operatorname{ggF}$ & $\mathrm{VBF}$ & $\mathrm{WH}$ & $\mathrm{ZH}$ & $\mathrm{ttH}$ \\
\hline experimental & 1.03 & 1.18 & 0.89 & 0.79 & 2.3 \\
error & 0.15 & 0.24 & 0.39 & 0.37 & 0.65 \\
theory & $\left(\frac{\epsilon c_{g g}}{c_{g g}^{S M}}\right)^{2}$ & $\epsilon^{2}$ & $\epsilon^{2}$ & $\epsilon^{2}$ & $\epsilon^{2}$ \\
\hline
\end{tabular}

Table 4.1: Signal strengths for Higgs boson production channels according to ATLAS/CMS collab. [30], including predictions from the model in terms of $\epsilon$ and $c_{\gamma \gamma}$

\begin{tabular}{lccccc}
\hline & $\gamma \gamma$ & ZZ & WW & $\tau \tau$ & bb \\
\hline experimental & 1.14 & 1.29 & 1.09 & 1.11 & 0.70 \\
error & 0.19 & 0.25 & 0.17 & 0.23 & 0.28 \\
theory & $\left(\frac{\epsilon c_{\gamma \gamma}}{c_{\gamma \gamma}^{S M}}\right)^{2}$ & $\epsilon^{2}$ & $\epsilon^{2}$ & $\epsilon^{2}$ & $\epsilon^{2}$ \\
\hline
\end{tabular}

Table 4.2: Signal strengths for Higgs boson decay channels according to ATLAS/CMS collab. [30], including predictions from the model in terms of $\epsilon$ and $c_{g g}$.

The total width of the dilaton is

$$
\Gamma_{t o t}=\epsilon^{2}\left(\sum_{f} \Gamma_{f}^{S M}+\Gamma_{W W}^{S M}+\Gamma_{Z Z}^{S M}+\Gamma_{\gamma Z}^{S M}+\left(\frac{c_{g g}}{c_{g g}^{S M}}\right)^{2} \Gamma_{g g}^{S M}+\left(\frac{c_{\gamma \gamma}}{c_{\gamma \gamma}^{S M}}\right)^{2} \Gamma_{\gamma \gamma}^{S M}\right)+\Gamma_{i n v}
$$

where $\Gamma_{i n v}$ is the invisible width of the process $\sigma \rightarrow S S$. The fit is done by a Markov Chain Monte Carlo (MCMC) algorithm, using a Metropolis-Hastings algorithm with simulated annealing [2]. There are 7 degrees of freedom derived from the 10 Higgs observables and the three parameters appearing in the dilaton couplings: $\epsilon, c_{g g}$, and $c_{\gamma \gamma}$. The target distribution is a Gaussian likelihood function:

$$
\mathcal{L}=\prod_{i} \exp \left[-\frac{\left(x_{i}-\bar{x}_{i}\right)^{2}}{2 \sigma_{i}^{2}}\right]
$$

where $x_{i}$ are the theoretical predictions, $\bar{x}_{i}$ are the experimental observables, and $\sigma_{i}$ are the errors on these observables.

There also exists a fit from electroweak precision data (EWPD) that places a limit 
on the value of $\epsilon$, by looking specifically at non-standard Higgs couplings. This places a lower bound on $\epsilon$ of about 0.98 [31].

First, we test the effectiveness of the fit by comparing with fits of a Higgs-like dilaton without the dark matter candidate. We note that our fit gives similar largeepsilon behaviour as that of previous work [29].

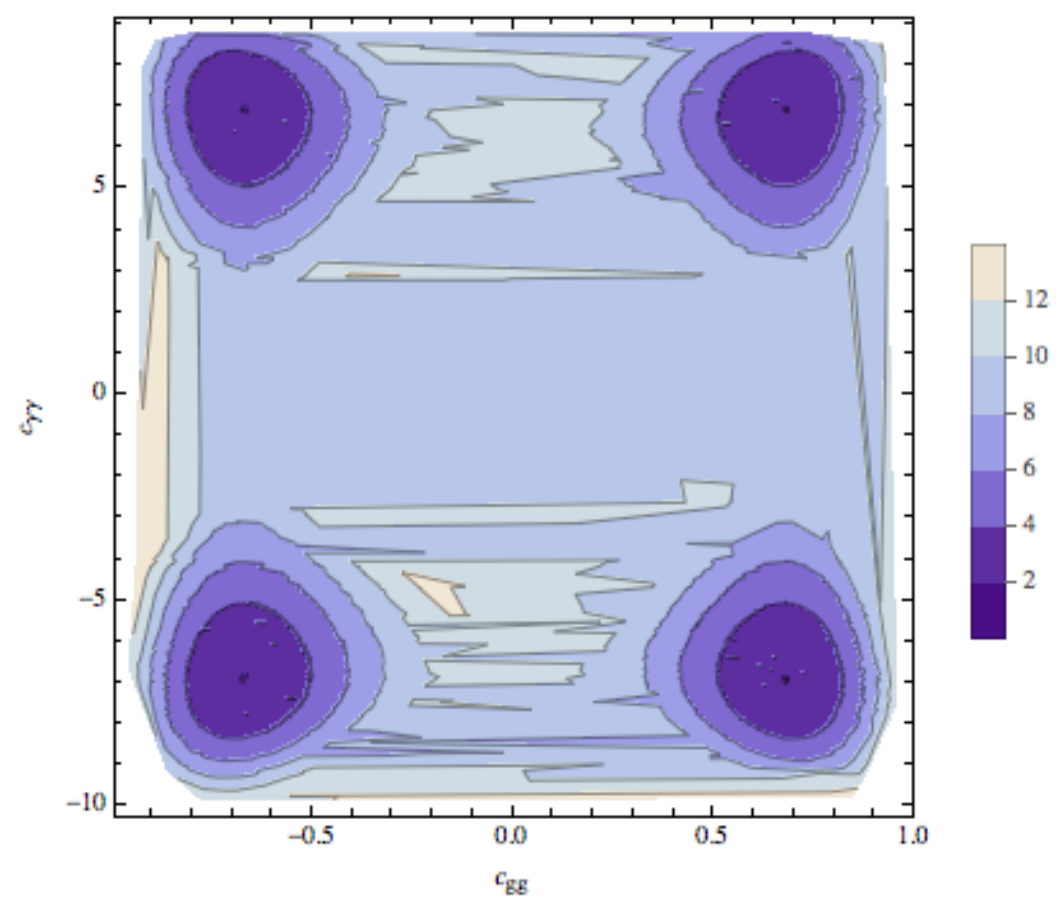

Figure 4.1: Parameter space of $c_{g g}$ and $c_{\gamma \gamma}$, where the allowed region is $\chi^{2}<5.9$.

The dark matter candidate is then included in the fitting procedure. The fit is further extended by including another observable related to the dark matter mass: the total width of the Higgs boson. The bound derived in Reference [32] is $6.7_{-2.9}^{+7.7}$ $\mathrm{MeV}$. This agrees with a CMS study that places an upper bound of $22 \mathrm{MeV}$ at $95 \%$ CL [33]. Figure 4.1 shows the results of the MCMC. The $95 \%$ confidence interval for seven degrees of freedom corresponds to a $\chi^{2}$ of 5.9. The parameter space at $95 \%$ confidence is $0.5 \leq\left|c_{g g}\right| \leq 1$, while $4 \leq\left|c_{\gamma \gamma}\right| \leq 8$. The fourfold symmetry comes from the quadratic dependance on $c_{g g}$ and $c_{\gamma \gamma}$. These compare well to the Standard Model values of $c_{g g}^{S M}=0.67$ and $c_{\gamma \gamma}^{S M}=-6.5$. It is notable that some regions of parameter space, where $m_{S}$ is less than half the dilaton mass, can result in a large contribution to invisible decays, discussed in the next section. 


\subsubsection{Higgs Invisible Width}

The decay of the dilaton to two dark matter candidates is kinematically accessible when $m_{S}<m_{\sigma} / 2$. In this model, the invisible decay width of the dilaton is given by

$$
\Gamma(\sigma \rightarrow S S)=\frac{m_{S}^{4} \epsilon^{2}}{8 \pi m_{\sigma} v^{2}} \sqrt{1-4 \frac{m_{S}^{2}}{m_{\sigma}^{2}}},
$$

where the branching ratio is analogous to Equation 3.3:

$$
B R(\sigma \rightarrow i n v .)=\frac{\Gamma(\sigma \rightarrow S S)}{\Gamma_{t o t}}
$$

with $\Gamma_{\text {tot }}$ defined in equation 4.5.

In Figure 4.2 the branching ratio is plotted against the dark matter mass, including the experimental upper bound from ATLAS/CMS of 0.28 [19].

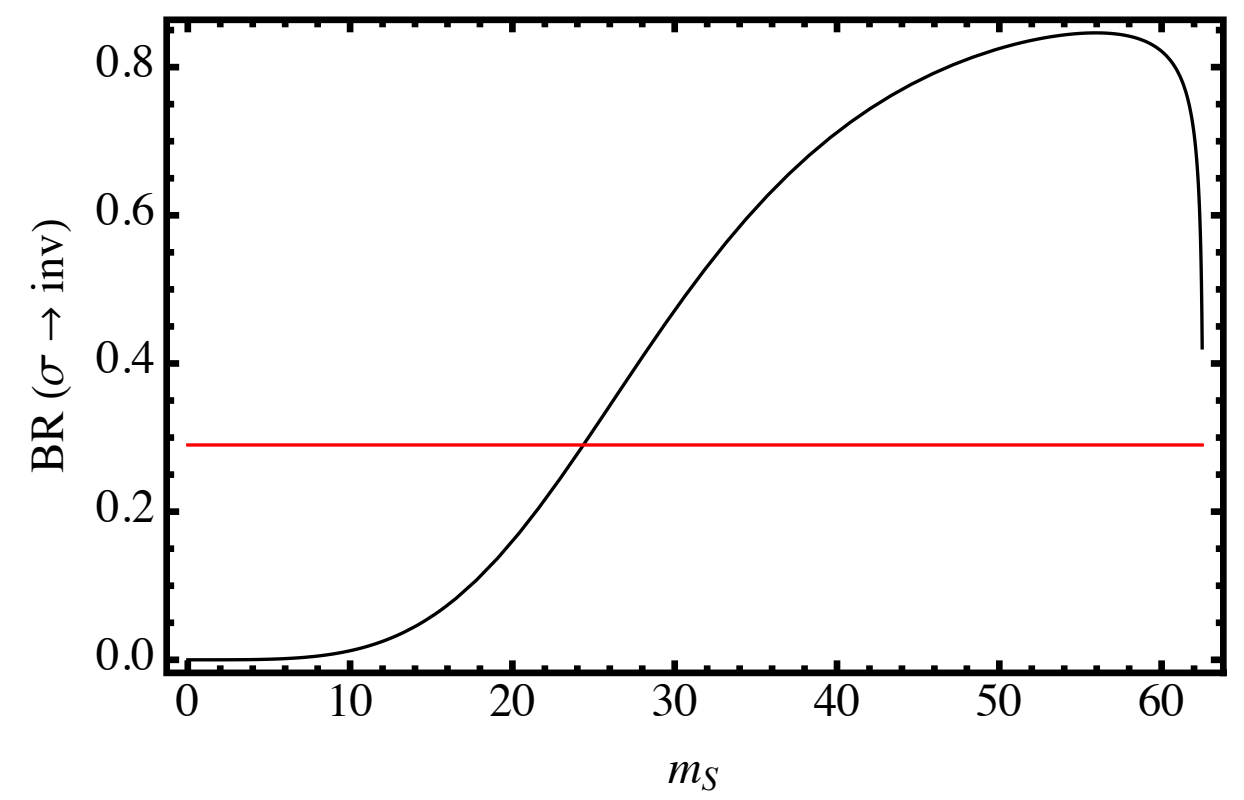

Figure 4.2: The branching ratio for the dilaton decay to invisible particles, with $c_{g g}=0.67$ and $c_{\gamma \gamma}=-6.5$. The horizontal line is the ATLAS upper bound [19].

The Standard Model values were used in Figure 4.2 for $c_{g g}$ and $c_{\gamma \gamma}$. Given the bounds from signal strengths found in the previous section, the constraint from the invisible width does not greatly depend on $c_{g g}$, and even less so on $c_{\gamma \gamma}$ given the mass of the DM. This is illustrated for the former case in Figure 4.3, where contours of 
the branching ratio is shown for varying $m_{S}$ and $c_{g g}$. Selecting a value of $m_{S}$ gives a number for the branching ratio almost completely independent of the value of $c_{g g}$.

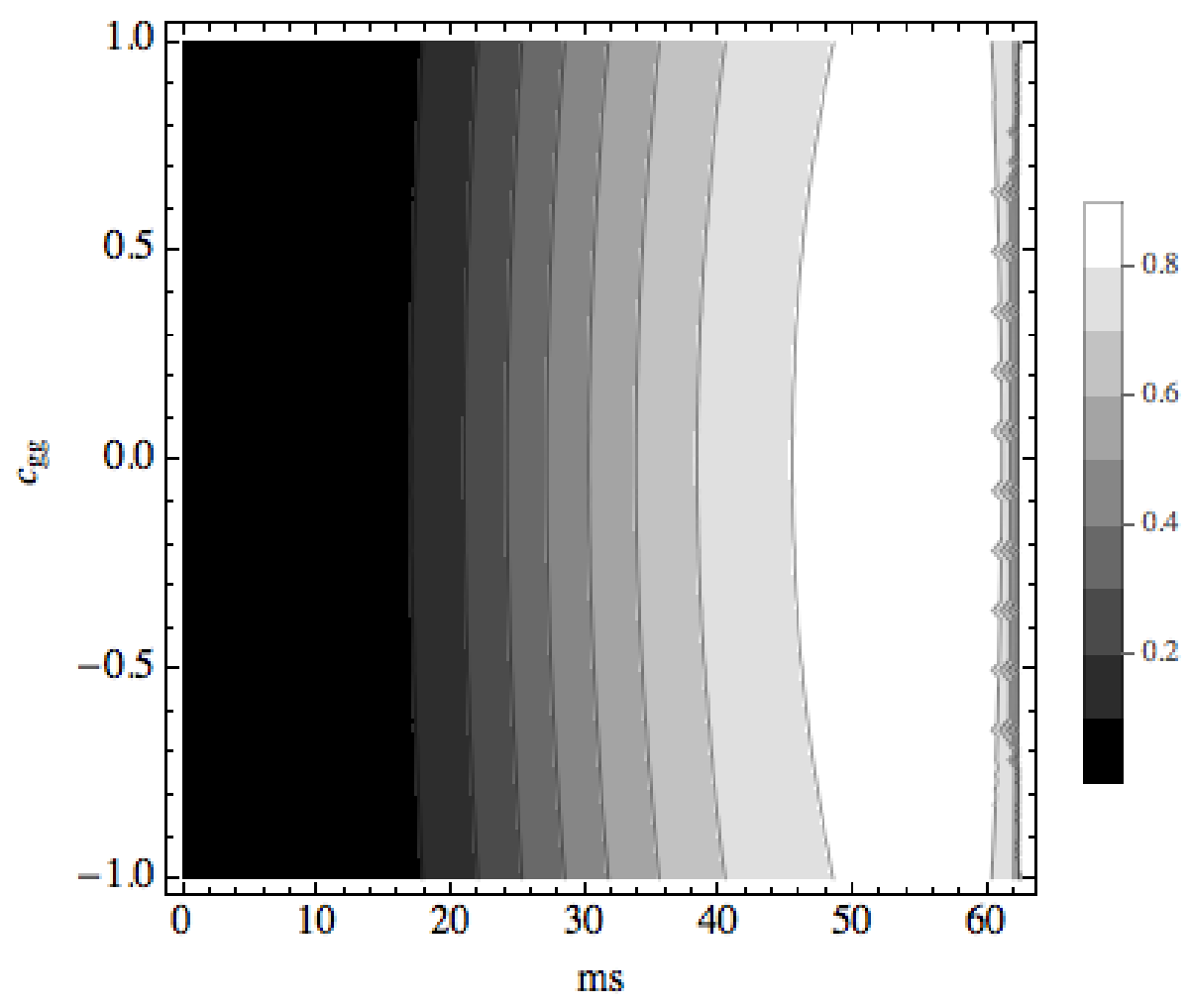

Figure 4.3: A contour plot of the $m_{S}$ and $c_{g g}$. The contours are the values for the branching ratio, with an experimental cutoff of 0.28 . The value of $c_{g g}$ is clearly not sensitive to the value of $m_{S}$ - no new information is gained concerning these parameters.

\subsubsection{Dark Matter Relic Abundance}

In order to calculate the relic abundance, all reactions of the form $\mathrm{SS} \rightarrow \mathrm{xx}$, where $\mathrm{x}$ is a standard model particle or the dilaton, must be taken into account for the thermalized cross section.

Using the Feynman rules in Appendix A, each annihilation cross section can be calculated individually. We start with the processes with SM particles in the final 

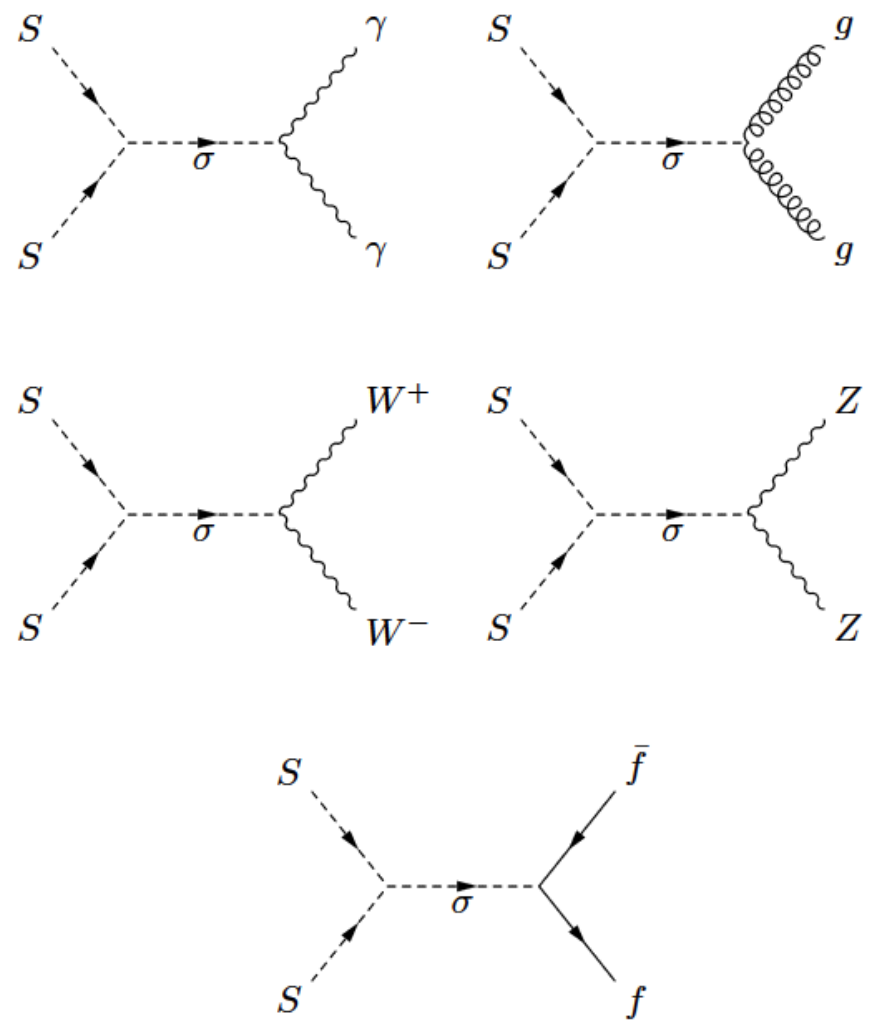

Figure 4.4: The Feynman diagrams for the processes $\mathrm{SS} \rightarrow \mathrm{xx}$, where $\mathrm{x}$ is a $\mathrm{SM}$ particle.

state, illustrated by Figure 4.4. For the process $S S \rightarrow \mathrm{ZZ}$,

$$
\sigma_{Z Z}(s)=\frac{1}{8 \pi} \frac{\sqrt{s-4 m_{Z}^{2}}}{\sqrt{s-4 m_{S}^{2}}}\left(\frac{m_{S} \epsilon}{v}\right)^{4} \frac{s}{\left(s-m_{\sigma}^{2}\right)^{2}+\Gamma_{\sigma}^{2} m_{\sigma}^{2}}\left(1+\frac{12 m_{Z}^{4}}{s^{2}}-\frac{4 m_{Z}^{2}}{s}\right)
$$

and similarly for $\mathrm{W}$ bosons in the final state,

$$
\sigma_{W W}(s)=\frac{1}{4 \pi} \frac{\sqrt{s-4 m_{W}^{2}}}{\sqrt{s-4 m_{S}^{2}}}\left(\frac{m_{S} \epsilon}{v}\right)^{4} \frac{s}{\left(s-m_{\sigma}^{2}\right)^{2}+\Gamma_{\sigma}^{2} m_{\sigma}^{2}}\left(1+\frac{12 m_{W}^{4}}{s^{2}}-\frac{4 m_{W}^{2}}{s}\right) .
$$

The annihilation cross section into photons is

$$
\sigma_{\gamma \gamma}(s)=\frac{\left(c_{\gamma \gamma} \alpha_{E M}\right)^{2}}{8 \pi^{3}} \frac{s}{\left(s-m_{\sigma}^{2}\right)^{2}-\Gamma_{\sigma}^{2} m_{\sigma}^{2}}\left(\frac{m_{S} \epsilon}{v}\right)^{4}\left(1-\frac{4 m_{S}^{2}}{s}\right)^{-\frac{1}{2}} .
$$


The annihilation cross section into gluons is

$$
\sigma_{g g}(s)=\frac{\left(c_{g g} \alpha_{S}\right)^{2}}{\pi^{3}} \frac{s}{\left(s-m_{\sigma}^{2}\right)^{2}-\Gamma_{\sigma}^{2} m_{\sigma}^{2}}\left(\frac{m_{S} \epsilon}{v}\right)^{4}\left(1-\frac{4 m_{S}^{2}}{s}\right)^{-\frac{1}{2}}
$$

The annihilation cross section to fermions is

$$
\sigma_{f \bar{f}}(s)=\frac{2 m_{f}^{2} N_{C}}{\pi s}\left(\frac{m_{S} \epsilon}{v}\right)^{4} \frac{1}{\left(s-m_{\sigma}^{2}\right)^{2}+\Gamma_{\sigma}^{2} m_{\sigma}^{2}} \frac{\left(s-4 m_{f}^{2}\right)^{\frac{3}{2}}}{\sqrt{s-4 m_{S}^{2}}}
$$

where $N_{C}=3$ for quarks, else $N_{C}=1$.

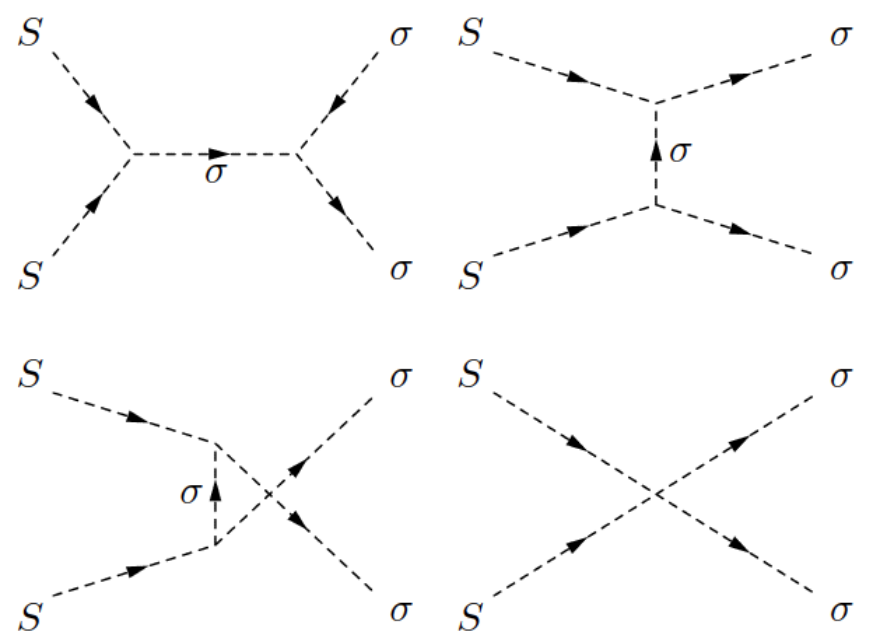

Figure 4.5: The Feynman diagrams for the process $\mathrm{SS} \rightarrow \sigma \sigma$. 
Finally, the annihilation cross section to dilatons must include four different Feynman diagrams, shown in Figure 4.5. The result is given by

$$
\begin{array}{r}
\sigma_{\sigma \sigma}(s)=\frac{\sqrt{s-4 m_{\sigma}^{2}}}{4 \pi s \sqrt{s-4 m_{S}^{2}}}\left(\frac{m_{S} \epsilon}{v}\right)^{4}\left(2-\frac{m_{S}^{4}}{m_{\sigma}^{2} m_{S}^{2}-\frac{1}{4}\left(m_{\sigma}^{4}-s m_{S}^{2}\right)}+\right. \\
\frac{3.38 m_{\sigma}^{4}-2 m_{\sigma}^{2}\left(m_{\sigma}^{2}-s\right)\left(3+\lambda_{p}\right)}{-m_{S}^{4}+\left(m_{\sigma}^{2}-s\right)^{2}+m_{\sigma}^{2} \Gamma_{\sigma}^{2}}+\frac{m_{S}^{4} \arctan \left(\frac{\sqrt{4 m_{\sigma}^{2}-s} \sqrt{s-4 m_{S}^{2}}}{2 m_{\sigma}^{2}-s}\right)}{2 \sqrt{1-\frac{4 m_{\sigma}^{2}}{s} \sqrt{1-\frac{4 m_{S}^{2}}{s}}\left(m_{\sigma}^{2}-s / 2\right) s}+} \\
\frac{m_{\sigma}^{2} m_{S}^{2}\left(m_{\sigma}^{2}-s\right)\left(3+\lambda_{p}\right)}{s\left(-m_{S}^{4}+\left(m_{\sigma}^{2}-s\right)^{2}+m_{\sigma}^{2} \Gamma_{\sigma}^{2}\right)} \log \left(\frac{2 m_{\sigma}^{2}+s\left(-1+\sqrt{1-\frac{4 m_{\sigma}^{2}}{s}} \sqrt{1-\frac{4 m_{S}^{2}}{s}}\right)}{2 m_{\sigma}^{2}-s}\right) \\
\left.\frac{8 m_{S}^{2} \log \left(\frac{2 m_{\sigma}^{2}+s\left(-1+\sqrt{1-\frac{4 m_{\sigma}^{2}}{s}} \sqrt{1-\frac{4 m_{S}^{2}}{s}}\right)}{2 m_{\sigma}^{2}-s}\right)}{}\right) \\
-
\end{array}
$$

The sum of the six processes is the total cross section, $\sigma(s)$. This can be inserted into Equation 3.6 to find the thermalized cross section, which is then used in Equation 3.5 to calculate the freeze-out temperature. Finally, evaluating the thermalized cross section at the freeze-out temperature and inserting into Equation 3.7 gives the relic abundance. This is compared with the observed relic abundance, $0.1199 \pm 0.0027$.

Figure 4.6 plots the present-day relic abundance against the DM mass, including the aforementioned experimental upper bound. The values for the relic abundance are checked using micrOMEGAs [34]. Included are the curves for $\lambda=3$, corresponding to SM Higgs self coupling, and $\lambda=4 \pi$, where strong interactions dominate. The model gives the observed DM relic abundance when mass $m_{S} \approx 50$, and rapidly becomes suppressed at masses close to the resonant region, $m_{\sigma} / 2$.

The singlet scalar dark matter candidate with a Higgs-like dilaton clearly cannot by itself reproduce the observed dark matter relic abundance. It can, however, give insight into the nature of the Higgs self-coupling. When dark matter annihilations into dilatons are kinematically allowed, the self coupling can dictate how dominant the decay is and therefore determine how large the DM abundance in that mass region 


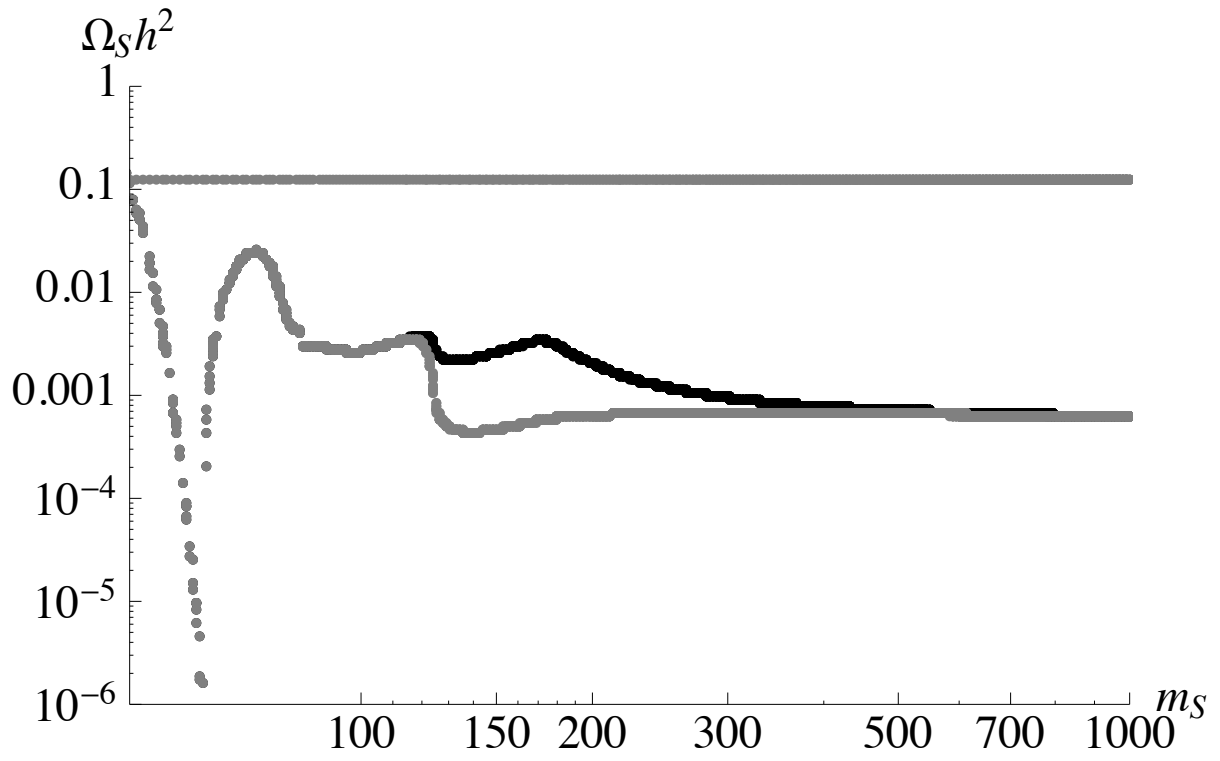

Figure 4.6: The DM relic abundance as a function of the DM mass. The $\lambda=3$ case corresponds to the black line, and the $\lambda=4 \pi$ case corresponds to the lower grey line. The horizontal grey line is the experimental value [21].

can be.

\subsubsection{Dark Matter Direct Detection}

We introduce the effective nucleon dilaton coupling $\mathrm{f}_{N}$, which can be written as a sum of the contribution from each quark coupling:

$$
f_{N}=\sum_{q}\langle N|\bar{q} q| N\rangle \frac{m_{q}}{m_{N}}=\sum_{q} f_{N}^{q}
$$

The contributions from $\mathrm{u}, \mathrm{d}$, and $\mathrm{s}$ are related to the light quark matrix elements, and values are taken from Reference [35] as follows:

$$
\begin{aligned}
& f_{N}^{u}=0.024 \\
& f_{N}^{d}=0.033 \\
& f_{N}^{s}=0.042 .
\end{aligned}
$$


These values along with the heavy quark contributions give the Standard Model value $f_{N}^{S M}=0.30$, which agrees with Reference [35]. Since this model subsumes the SM heavy quark contribution into $c_{g g}$, there must be a correction to $f_{N}$ by the gluon contribution

$$
f_{N}=\epsilon\left(f^{u}+f^{d}+f^{s}+f^{G}\right)=\epsilon\left(0.099+0.201 \frac{c_{g g}}{c_{g g}^{S M}}\right) .
$$

This expression is used to calculate the direct detection cross section:

$$
\sigma(N S \rightarrow N S)=\left(\frac{m_{N} m_{S}}{m_{\sigma} v}\right)^{4} \frac{\epsilon^{2} f_{N}^{2}}{\pi\left(m_{N}+m_{S}\right)^{2}},
$$

where $\mathrm{m}_{N}$ is the nucleon mass $(938.95 \mathrm{MeV})$.

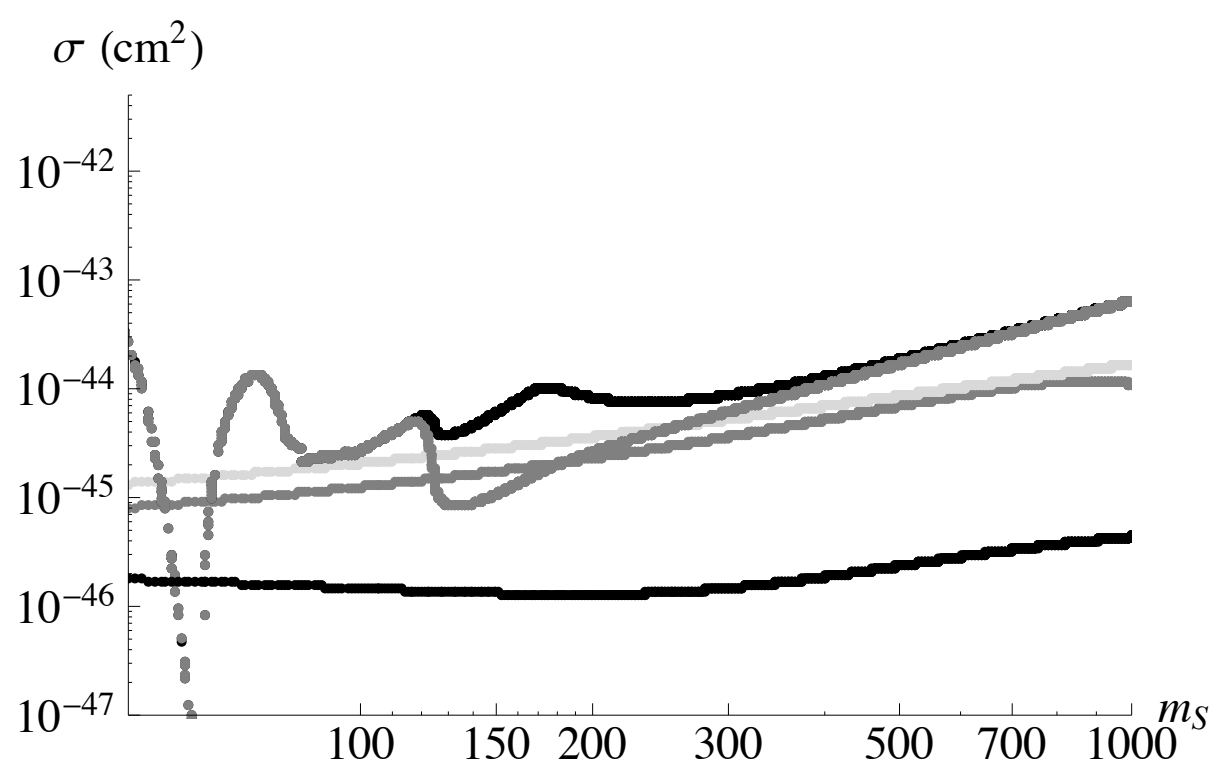

Figure 4.7: The DM direct detection cross section as a function of the DM mass. The cases for $\lambda=3$ and $\lambda=4 \pi$ are shown in black and dark grey, respectively. The experimental limits are, starting from bottom: DEAP projection (black), LUX (grey), and XENON (light grey).

The direct detection cross section is shown in Figure 4.7 as a function of $m_{S}$. Included are the experimental limits from XENON [23], LUX [24], and the projected limits from DEAP 3600 [27], all of which were extracted using Reference [36]. Note that although negative values of $c_{g g}$ are allowed from the signal strength fit as shown in Figure 4.1, the points included were obtained using $c_{g g}>0$. It is interesting to 
note that some of the points allowed by LUX and XENON would be ruled out by the DEAP expected limit.

\subsection{Summary}

A model is introduced that treats the SM physical Higgs boson as a pseudoGoldstone boson of spontaneous conformal symmetry breaking, the dilaton, and introduces a singlet scalar dark matter candidate. The relevant model parameters are fitted to the measured Higgs signal strengths, which restricts $c_{g g}$ and $c_{\gamma \gamma}$ to a parameter space consistent with the SM values. The relic abundance and direct detection constraints limit the possible DM masses to a small range, but is increased to the range $125-300 \mathrm{GeV}$ when the self coupling is changed to be that of a strongly interacting composite region. In addition, the non-linear nature of the coupling between the dilaton and $S$ yield large couplings for masses $m_{S}<m_{\sigma} / 2$ and thus DM candidates below this limit are ruled out by the current upper limit of the Higgs invisible width. 


\section{Chapter 5}

\section{Georgi-Machacek Model with Scalar DM}

\subsection{Background and Motivation}

A natural step for BSM model building is to start with the existing SM framework and add extra doublets or triplets. The Georgi-Machacek (GM) Model is a wellestablished benchmark model that features an extended Higgs sector and preserves custodial SU(2) symmetry. This Higgs sector consists of a doublet, real triplet, and complex triplet [37]. This chapter studies the GM model extended by a singlet scalar dark matter candidate (SSDM), S. It is assumed for simplicity that S accounts for all of the observed dark matter relic abundance via the standard thermal freeze-out mechanism. This work is currently in preparation for publication [3].

\section{The Georgi-Machacek Model}

The scalar sector of the GM model ${ }^{1}$ consists of the usual complex Higgs doublet $\left(\phi^{+}, \phi^{0}\right)$, a real triplet $\left(\xi^{+}, \xi^{0}, \xi^{-}\right)$, and a complex triplet $\left(\chi^{++}, \chi^{+}, \chi^{0}\right)$. The doublet functions as in the SM Higgs mechanism. This doublet can be written in the form of a bidoublet $\Phi$ and the triplets are combined to form a bitriplet $X$ :

$$
\Phi=\left(\begin{array}{cc}
\phi^{0 *} & \phi^{+} \\
-\phi^{+*} & \phi^{0}
\end{array}\right), \quad X=\left(\begin{array}{ccc}
\chi^{0 *} & \xi^{+} & \chi^{++} \\
-\chi^{+*} & \xi^{0} & \chi^{+} \\
\chi^{++*} & -\xi^{+*} & \chi^{0}
\end{array}\right)
$$

The vevs are defined by $\langle\Phi\rangle=\frac{v_{\phi}}{\sqrt{2}} I_{2 \times 2}$ and $\langle X\rangle=v_{\chi} I_{3 \times 3}$, where $I$ is the unit

\footnotetext{
${ }^{1}$ For a more detailed introduction to the Georgi-Machacek model, see Reference [38].
} 
matrix and the Fermi constant $G_{F}$ constrains

$$
v_{\phi}^{2}+8 v_{\chi}^{2} \equiv v^{2}=\frac{1}{\sqrt{2} G_{F}} \approx(246 \mathrm{GeV})^{2}
$$

The most general gauge-invariant scalar potential involving these fields and the real singlet $S$, while conserving the global $\mathrm{SU}(2)_{L} \times \mathrm{SU}(2)_{R}$ and the $Z_{2}$ symmetry $S \rightarrow-S$, is given by

$$
\begin{aligned}
V(\Phi, X)= & \frac{\mu_{2}^{2}}{2} \operatorname{Tr}\left(\Phi^{\dagger} \Phi\right)+\frac{\mu_{3}^{2}}{2} \operatorname{Tr}\left(X^{\dagger} X\right)+\lambda_{1}\left[\operatorname{Tr}\left(\Phi^{\dagger} \Phi\right)\right]^{2}+\lambda_{2} \operatorname{Tr}\left(\Phi^{\dagger} \Phi\right) \operatorname{Tr}\left(X^{\dagger} X\right) \\
& +\lambda_{3} \operatorname{Tr}\left(X^{\dagger} X X^{\dagger} X\right)+\lambda_{4}\left[\operatorname{Tr}\left(X^{\dagger} X\right)\right]^{2}-\lambda_{5} \operatorname{Tr}\left(\Phi^{\dagger} \tau^{a} \Phi \tau^{b}\right) \operatorname{Tr}\left(X^{\dagger} t^{a} X t^{b}\right) \\
& -M_{1} \operatorname{Tr}\left(\Phi^{\dagger} \tau^{a} \Phi \tau^{b}\right)\left(U X U^{\dagger}\right)_{a b}-M_{2} \operatorname{Tr}\left(X^{\dagger} t^{a} X t^{b}\right)\left(U X U^{\dagger}\right)_{a b} \\
& +\frac{\mu_{S}^{2}}{2} S^{2}+\lambda_{a} S^{2} \operatorname{Tr}\left(\Phi^{\dagger} \Phi\right)+\lambda_{b} S^{2} \operatorname{Tr}\left(X^{\dagger} X\right)+\lambda_{S} S^{4} .
\end{aligned}
$$

The last line of the potential in Equation 5.3 contains the terms for the singlet scalar DM, while the rest is from the GM model. The physical fields that emerge can be organized by their transformation properties under the custodial $\mathrm{SU}(2)$ symmetry into a fiveplet, a triplet, and three singlets, one of which is $S$. The fiveplet and triplet states are given by (plus the hermitian conjugate where appropriate)

$$
\begin{aligned}
& H_{5}^{++}=\chi^{++}, \quad H_{5}^{+}=\frac{\left(\chi^{+}-\xi^{+}\right)}{\sqrt{2}}, \quad H_{5}^{0}=\sqrt{\frac{2}{3}} \xi^{0}-\sqrt{\frac{1}{3}} \chi^{0, r}, \\
& H_{3}^{+}=-s_{H} \phi^{+}+c_{H} \frac{\left(\chi^{+}+\xi^{+}\right)}{\sqrt{2}}, \quad H_{3}^{0}=-s_{H} \phi^{0, i}+c_{H} \chi^{0, i}
\end{aligned}
$$

where the vevs are parameterized by

$$
c_{H} \equiv \cos \theta_{H}=\frac{v_{\phi}}{v}, \quad s_{H} \equiv \sin \theta_{H}=\frac{2 \sqrt{2} v_{\chi}}{v}
$$

and the neutral fields are decomposed into real and imaginary parts according to

$$
\phi^{0} \rightarrow \frac{v_{\phi}}{\sqrt{2}}+\frac{\phi^{0, r}+i \phi^{0, i}}{\sqrt{2}}, \quad \chi^{0} \rightarrow v_{\chi}+\frac{\chi^{0, r}+i \chi^{0, i}}{\sqrt{2}}, \quad \xi^{0} \rightarrow v_{\chi}+\xi^{0} .
$$


The masses within each custodial multiplet can be written in terms of the vevs as

$$
\begin{aligned}
& m_{5}^{2}=\frac{M_{1}}{4 v_{\chi}} v_{\phi}^{2}+12 M_{2} v_{\chi}+\frac{3}{2} \lambda_{5} v_{\phi}^{2}+8 \lambda_{3} v_{\chi}^{2} \\
& m_{3}^{2}=\frac{M_{1}}{4 v_{\chi}}\left(v_{\phi}^{2}+8 v_{\chi}^{2}\right)+\frac{\lambda_{5}}{2}\left(v_{\phi}^{2}+8 v_{\chi}^{2}\right)=\left(\frac{M_{1}}{4 v_{\chi}}+\frac{\lambda_{5}}{2}\right) v^{2} .
\end{aligned}
$$

The gauge singlet $S$ remains a mass eigenstate, with physical mass-squared given by

$$
m_{S}^{2}=\mu_{S}^{2}+2 \lambda_{a} v_{\phi}^{2}+6 \lambda_{b} v_{\chi}^{2}
$$

which is required to be positive to avoid breaking the $Z_{2}$ symmetry.

The other mass eigenstates are given by

$$
h=\cos \alpha \phi^{0, r}-\sin \alpha H_{1}^{0 \prime}, \quad H=\sin \alpha \phi^{0, r}+\cos \alpha H_{1}^{0 \prime},
$$

where

$$
H_{1}^{0 \prime}=\sqrt{\frac{1}{3}} \xi^{0}+\sqrt{\frac{2}{3}} \chi^{0, r} .
$$

The mixing angle and masses are given by

$$
\begin{aligned}
& \sin 2 \alpha=\frac{2 \mathcal{M}_{12}^{2}}{m_{H}^{2}-m_{h}^{2}}, \quad \cos 2 \alpha=\frac{\mathcal{M}_{22}^{2}-\mathcal{M}_{11}^{2}}{m_{H}^{2}-m_{h}^{2}}, \\
& m_{h, H}^{2}=\frac{1}{2}\left[\mathcal{M}_{11}^{2}+\mathcal{M}_{22}^{2} \mp \sqrt{\left(\mathcal{M}_{11}^{2}-\mathcal{M}_{22}^{2}\right)^{2}+4\left(\mathcal{M}_{12}^{2}\right)^{2}}\right],
\end{aligned}
$$

where the definition $m_{h}<m_{H}$ is chosen, and

$$
\begin{aligned}
\mathcal{M}_{11}^{2} & =8 \lambda_{1} v_{\phi}^{2} \\
\mathcal{M}_{12}^{2} & =\frac{\sqrt{3}}{2} v_{\phi}\left[-M_{1}+4\left(2 \lambda_{2}-\lambda_{5}\right) v_{\chi}\right] \\
\mathcal{M}_{22}^{2} & =\frac{M_{1} v_{\phi}^{2}}{4 v_{\chi}}-6 M_{2} v_{\chi}+8\left(\lambda_{3}+3 \lambda_{4}\right) v_{\chi}^{2} .
\end{aligned}
$$

The Feynman rules for this model are listed in Appendix A. To summarize, the GM+SSDM model has a total of 13 free parameters: $\lambda_{a}, \lambda_{b}, \lambda_{1}, \lambda_{2}, \lambda_{3}, \lambda_{4}, \lambda_{5}, \lambda_{S}$, $\mathrm{M}_{1}, \mathrm{M}_{2}, \mu_{2}^{2}, \mu_{3}^{2}$, and $\mu_{S}^{2}$. These will be constrained in the following section. 


\subsection{Phenomenology}

This section will look at the constraints on the parameters of the GM+SSDM model. First, theoretical constraints are applied. Then, we include experimental constraints from relic density, direct detection, Higgs invisible width and Higgs signal strengths. A plot in a given section will cumulatively include constraints from all those imposed before the constraints added in the section.

\subsubsection{Theoretical Constraints}

A number of theoretical constraints can be applied to the singlet scalar DM extension of the GM model. Firstly, perturbative unitarity imposed on the $2 \rightarrow 2$ scattering amplitudes is applied, then the vacuum stability constraints (meaning the potential is bounded from below), then conditions to avoid false minima. For more information, see References [3,39]. Experimental constraints applied to the generated points but not included in the body of this thesis are the limit on $b \rightarrow s \gamma$ and dwarf spheroidal galaxy constraints [38].

\subsubsection{Dark Matter Relic Density}

To calculate the relic density, the dark matter annihilation cross sections is considered for all possible processes. The annihilation into non-scalar SM particles $(\mathrm{A}, \mathrm{B})$ (Figure 5.1), gives a thermalized cross section of

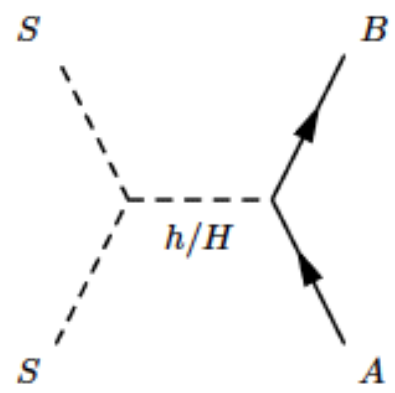

Figure 5.1: Feynman diagram for $S S \rightarrow A+B$ where A, B are SM particles 


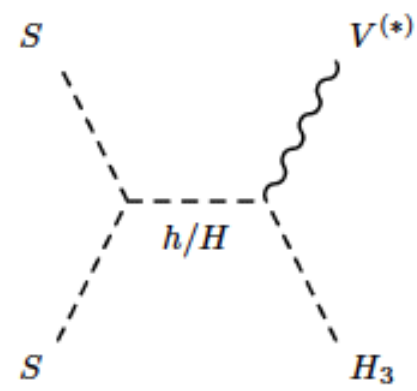

Figure 5.2: Feynman diagram for $S S \rightarrow H_{3} V^{(*)}$.

$$
\begin{aligned}
\sigma v_{\text {rel }}(S S \rightarrow A & +B)=\frac{4}{\sqrt{s}}\left(\frac{g_{S S h} g_{h A B}}{s-m_{h}^{2}}+\frac{g_{S S H} g_{H A B}}{s-m_{H}^{2}}\right)^{2} \\
& \times \frac{1}{g_{h_{S M}}^{2} A B} \Gamma\left(m_{h_{S M}}=\sqrt{s}, h_{S M} \rightarrow A+B\right)
\end{aligned}
$$

where $\Gamma$ is the partial width for the Higgs decaying into SM particles, evaluated when $m_{h_{S M}}=\sqrt{s}$. We also must include annihilation products of one scalar and one SM vector boson, and also the final state $V^{*} H_{3}$, where the gauge boson is off-shell (Figure 5.2). The thermalized cross section for the first process is

$$
\begin{array}{r}
\sigma v_{\mathrm{rel}}\left(S S \rightarrow H_{3} V\right)=\frac{\left(s^{2}-2 s\left(m_{H_{3}}^{2}+m_{V}^{2}\right)+\left(m_{H_{3}}^{2}-m_{V}^{2}\right)^{2}\right)^{\frac{3}{2}}}{8 \pi s^{2} m_{W}^{2}} \\
\times\left(\frac{g_{S S h} g_{h H_{3} V}}{s-m_{h}^{2}}+\frac{g_{S S H} g_{H H_{3} V}}{s-m_{H}^{2}}\right)^{2}
\end{array}
$$

while that for the second is [40]

$$
\sigma v_{\mathrm{rel}}\left(S S \rightarrow H_{3} V^{*}\right)=\left(\frac{g_{S S h} g_{h H_{3} V}}{s-m_{h}^{2}}+\frac{g_{S S H} g_{H H_{3} V}}{s-m_{H}^{2}}\right)^{2} \delta_{V} \frac{3 m_{V}^{2}}{4 \pi^{3} v^{2}} G_{i j}
$$


where

$$
\begin{aligned}
G_{i j}= & \frac{1}{4}\left(2\left(-1+k_{j}-k_{i}\right) \sqrt{\lambda_{i j}}\left(\frac{\pi}{2}+\arctan \left(\frac{k_{j}\left(1-k_{j}+k_{i}\right)-\lambda_{i j}}{\left(1-k_{i}\right) \sqrt{\lambda_{i j}}}\right)\right)\right. \\
& \left.+\left(\lambda_{i j}-2 k_{i}\right) \log k_{i}+\frac{1}{3}\left(1-k_{i}\right)\left(5\left(1+k_{i}\right)-4 k_{j}+\frac{2 \lambda_{i j}}{k_{j}}\right)\right) \\
\delta_{W}= & \frac{3}{2} \\
\delta_{Z}= & 3\left(\frac{7}{12}-\frac{10}{9} s_{W}^{2}+\frac{40}{27} s_{W}^{4}\right) .
\end{aligned}
$$

Here, $k_{i}=m_{H_{3}}^{2} / s, k_{j}=m_{V}^{2} / s$, and $\lambda_{i j}=-1+2 k_{i}+2 k_{j}-\left(k_{i}-k_{j}\right)^{2}$.
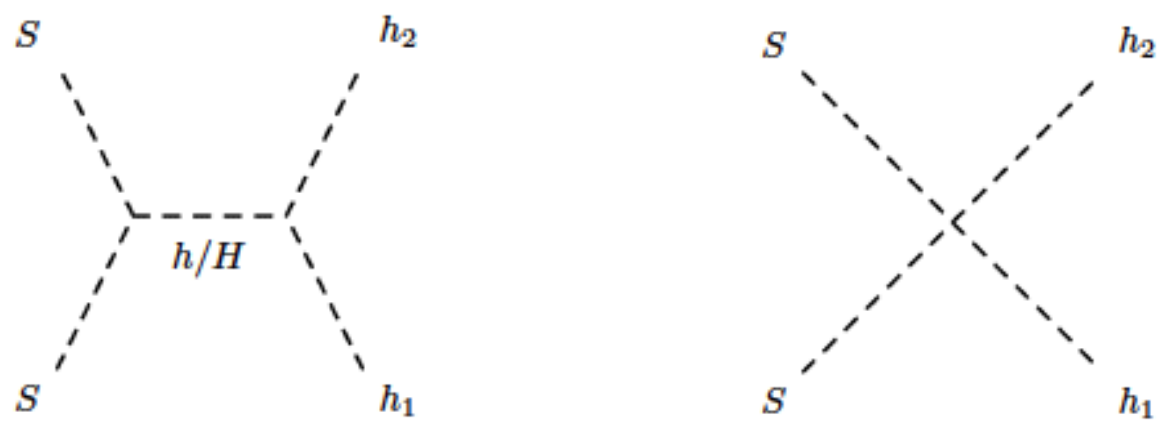

Figure 5.3: Feynman diagrams for $S S \rightarrow h_{1} h_{2}$

Next we consider DM annihilation into any of the following combinations of the Higgs triplet scalar fields:

$$
\left(H_{3}^{0}, H_{3}^{0}\right),\left(H_{3}^{+}, H_{3}^{-}\right),\left(H_{5}^{0}, H_{5}^{0}\right),\left(H_{5}^{+}, H_{5}^{-}\right),\left(H_{5}^{++}, H_{5}^{--}\right) .
$$

In this case we must take into account an additional four-point diagram.

The cross section for identical particles is given by:

$$
\sigma_{C M} v_{\text {rel }}\left(S S \rightarrow h_{1} h_{2}\right)=\frac{\left(s-4 m_{h_{1}}^{2}\right)^{\frac{1}{2}}}{32 \pi s^{\frac{3}{2}}}\left(g_{S S h_{1} h_{2}}+\frac{g_{S S h} g_{h h_{1} h_{2}}}{s-m_{h}^{2}}+\frac{g_{S S H} g_{H h_{1} h_{2}}}{s-m_{H}^{2}}\right)^{2}
$$

and for non-identical particles:

$$
\sigma_{C M} v_{\mathrm{rel}}\left(S S \rightarrow h_{1} h_{2}\right)=\frac{\left(s-4 m_{h_{1}}^{2}\right)^{\frac{1}{2}}}{16 \pi s^{\frac{3}{2}}}\left(g_{S S h_{1} h_{2}}+\frac{g_{S S h} g_{h h_{1} h_{2}}}{s-m_{h}^{2}}+\frac{g_{S S H} g_{H h_{1} h_{2}}}{s-m_{H}^{2}}\right)^{2}
$$


where $h_{1}$ and $h_{2}$ are any of the scalar pairs listed in Eqn. 5.19.
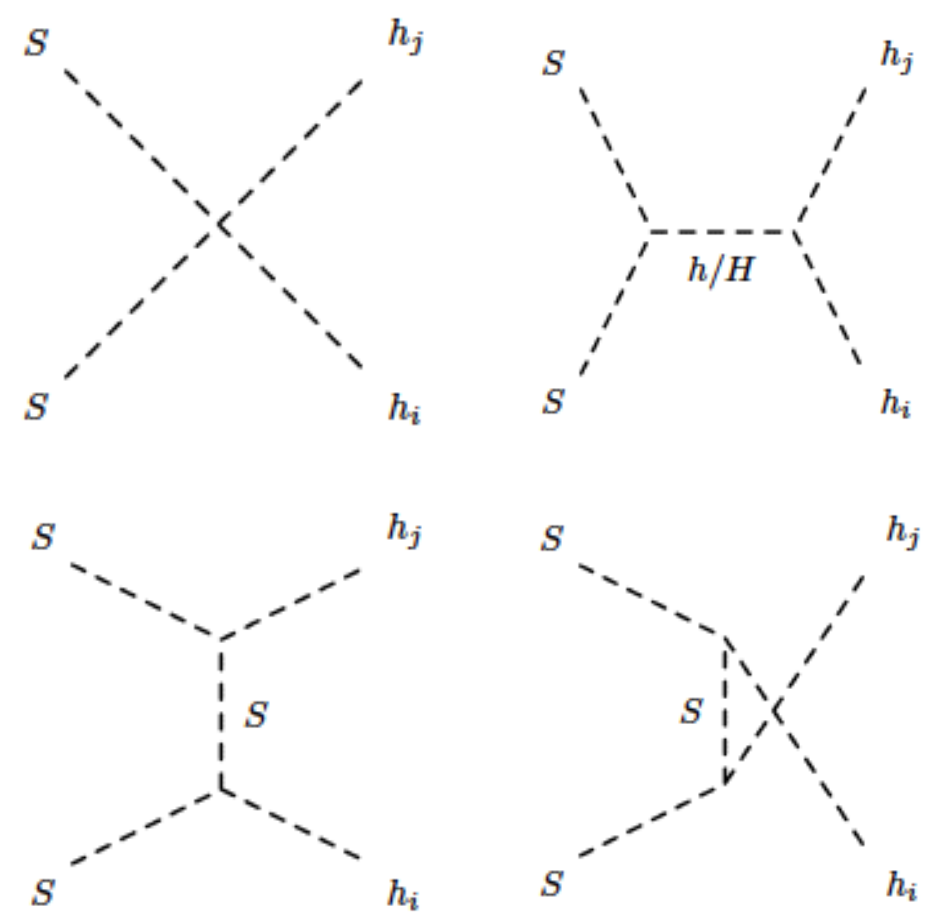

Figure 5.4: Feynman diagrams for process $S S \rightarrow h_{i} h_{j}$

The cross sections with $(h, H),(h, h)$ or $(H, H)$ in the final state include an additional t-channel and u-channel diagram as well as the s-channel and the four point interaction in Figure 5.4. For non identical $h_{i}, h_{j}$ in the final state, the end result is

$$
\frac{d \sigma}{d \Omega} v_{r e l}=\frac{\sqrt{s^{2}+\left(m_{h_{j}}^{2}-m_{h_{i}}^{2}\right)^{2}-2 s\left(m_{h_{j}}^{2}+m_{h_{i}}^{2}\right)}}{32 \pi^{2} s^{2}}\left[a+\frac{b}{c^{2}-\cos ^{2}(\theta) d^{2}}\right]^{2}
$$

where we define for convenience

$$
\begin{aligned}
a & =g_{S S h_{i} h_{j}}+\frac{g_{S S h_{i}} g_{h_{i} h_{i} h_{j}}}{s-m_{h_{i}}^{2}}+\frac{g_{S S h_{j}} g_{h_{i} h_{j} h_{j}}}{s-m_{h_{j}}^{2}} \\
b & =4 g_{S S h_{i}} g_{S S h_{j}}\left(s-\left(m_{h_{i}}^{2}+m_{h_{j}}^{2}\right)\right) \\
c^{2} & =\left(s-m_{h_{j}}^{2}-m_{h_{i}}^{2}\right)^{2} \\
d^{2} & =\left(1-\frac{4 m_{S}^{2}}{s}\right)\left(\left(s-m_{h_{j}}^{2}+m_{h_{i}}^{2}\right)^{2}-4 s m_{h_{i}}^{2}\right)
\end{aligned}
$$


and

$g(s)=\int_{-1}^{1} d \cos (\theta)\left[a-\frac{b}{c^{2}-\cos ^{2}(\theta) d^{2}}\right]^{2}=2 a^{2}+\frac{b^{2}}{c^{2}\left(c^{2}-d^{2}\right)}+\frac{b\left(b-4 a c^{2}\right)}{c^{3} d} \tanh ^{-1}\left(\frac{d}{c}\right)$.

For non identical $h_{i}, h_{j}$, the cross section is

$$
\sigma v_{r e l}=\frac{\sqrt{s^{2}+\left(m_{h_{j}}^{2}-m_{h_{i}}^{2}\right)^{2}-2 s\left(m_{h_{j}}^{2}+m_{h_{i}}^{2}\right)}}{16 \pi s^{2}} g(s)
$$

and for identical particles, is

$$
\sigma v_{r e l}=\frac{\sqrt{s-4 m_{h_{i}}^{2}}}{32 \pi s^{3 / 2}} g(s)
$$

This is applied in a slightly different way than that of Section 4.2.3. The thermalized cross section is calculated using equation 3.3 as used for the dilaton. We use the thermally averaged total annihilation cross section as input for the usual Boltzmann equation:

$$
\frac{d n_{S}}{d t}+3 H n_{S}=-\langle\sigma v\rangle\left[n_{S}^{2}-\left(n_{S}^{\mathrm{eq}}\right)^{2}\right]
$$

We solve this equation numerically to obtain the number density $n_{S}$ today which translates to a value for the relic abundance by multiplying by $m_{S}$ and dividing by the critical density of the universe today.

The relic density is a constraint on a combination of $\lambda_{a}$ and $\lambda_{b}$. To implement this, we first note that with all other parameters fixed, there is an oval like shaped set of $\left(\lambda_{a}, \lambda_{b}\right)$ for which we obtain the correct relic density. In the spirit of the numerical scans, we would like to be able to randomly select one such point. This is done by randomly generating an angle $\theta_{\lambda}$ in $\left[-\frac{\pi}{2}, \frac{\pi}{2}\right]$. Then we set $\lambda_{b}=\lambda_{a} \tan \theta_{\lambda}$, generate a mass $m_{S}$, and find the $\lambda_{a}$ that gives the correct relic abundance, which also in turn gives $\mu_{S}$. Searching for the correct value of $\lambda_{a}$ can be done easily since $\sigma v_{\text {rel }}$ is an increasing function of $\lambda_{a}$ and $\sigma v_{\text {rel }} \rightarrow \infty$ when $\lambda_{a} \rightarrow \infty$ and $\sigma v_{\text {rel }} \rightarrow \infty$ when $\lambda_{a} \rightarrow 0$. 


\subsubsection{Dark Matter Direct Detection}

In our model, direct detection proceeds via exchange of a virtual $h$ or $H$, as shown in Figure 5.5.

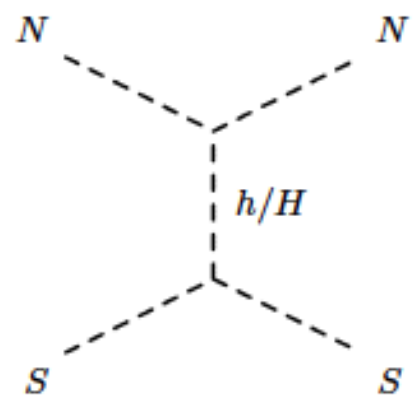

Figure 5.5: Feynman diagram for direct detection

The spin independent direct detection cross section is

$$
\sigma=\left(\frac{f_{N} g_{S S h} \cos (\alpha)}{m_{h}^{2} \cos \left(\theta_{H}\right)}+\frac{f_{N} g_{S S H} \sin (\alpha)}{m_{H}^{2} \cos \left(\theta_{H}\right)}\right)^{2} \frac{m_{N}^{4}}{4 \pi\left(m_{N}+m_{S}\right)^{2} v^{2}}
$$

where $\mathrm{f}_{N}$ is the nucleon vertex factor,

$$
f_{N}=\sum_{q} f_{q}=\sum_{q} \frac{m_{q}}{m_{N}}<N|\bar{q} q| N>
$$

and the sum is over all quark flavours. The value of $\mathrm{f}_{N}$ used is $0.30 \pm 0.03$, and the nucleon mass is $\mathrm{m}_{N}=938.95 \mathrm{MeV}$ [35]. The upper limits on the DM-Higgs vertices $\mathrm{g}_{S S h}$ and $\mathrm{g}_{S S H}$ were calculated in a previous paper [1]. Limits are included from the experiments LUX and XENON, as well as projected limits from DEAP $3600[23,24,27,36]$. Results are shown in Figure 5.6 for both the low and high mass regions. Points which fall above the experimental line are excluded, therefore direct detection effectively eliminates much of the low mass region. 

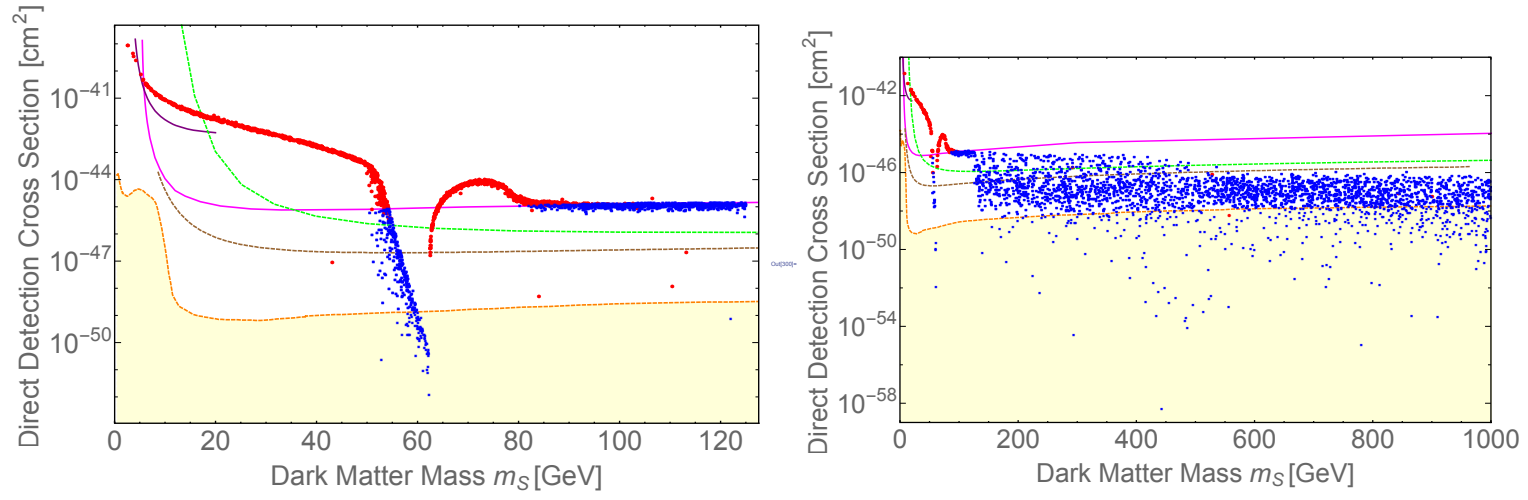

Figure 5.6: Direct detection cross section as a function of $m_{S}$. The left plot is a zoom of the low- $m_{S}$ region while the right plot shows the full $m_{S}$ range scanned. Experimental bounds include LUX (magenta), XENON10 (purple), XENON1T (brown), DEAP3600 (green), and the neutrino floor (yellow).

\subsubsection{Dark Matter Invisible Width}

When $m_{S}<m_{h, H} / 2$, the decay of the Higgs boson to two dark matter candidates is kinematically accessible. The expression for the width of this process is

$$
\Gamma_{S S h}=\frac{g_{S S h}^{2}}{32 m_{h}^{2} \pi} \sqrt{m_{h}^{2}-4 m_{s}^{2}} .
$$

To compare with experiment, the ratio

$$
\frac{\sigma_{V B F} B R_{i n v}}{\sigma_{V B F}^{S M}}=\frac{\left(\kappa_{V}\right)^{2} \Gamma(h \rightarrow i n v)}{\Gamma_{t o t}}
$$

is considered, written in terms of the vector boson fusion (VBF) production cross section and the invisible branching ratio, where $\kappa_{V}$ is defined in Equation 5.30. Here,

$$
\begin{aligned}
\Gamma_{t o t}= & \left(\kappa_{f}^{h}\right)^{2}\left[\Gamma(h \rightarrow b \bar{b})+\Gamma(h \rightarrow c \bar{c})+\Gamma\left(h \rightarrow \tau^{+} \tau^{-}\right)+\Gamma(h \rightarrow t \bar{t})\right] \\
& +\left(\kappa_{W}^{h}\right)^{2} \Gamma\left(h \rightarrow W W^{*}\right)+\left(\kappa_{Z}^{h}\right)^{2} \Gamma\left(h \rightarrow Z Z^{*}\right)+\Gamma(h \rightarrow \text { invs })
\end{aligned}
$$

The ratio in Equation 5.27 is shown in Figure 5.7, plotted against $\mathrm{m}_{S}$ in the kinematically allowed region. The experimental limit $[19,41]$ is the horizontal solid blue line, and excludes much of the remaining low mass DM parameter space. 


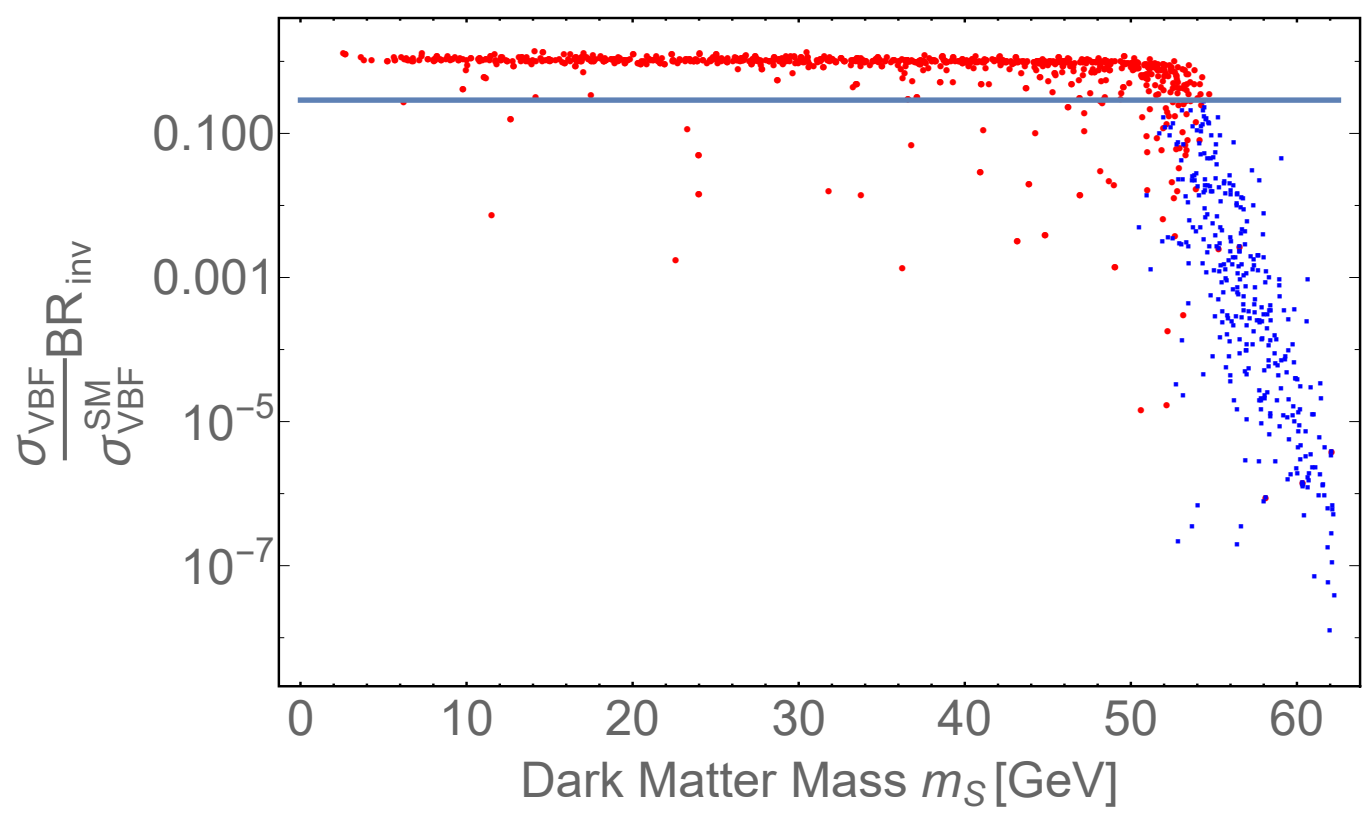

Figure 5.7: Constraint from invisible decays of the Higgs for a dark matter mass range between $0 \mathrm{GeV}$ and $125 / 2 \mathrm{GeV}$. The blue points are allowed while the red points are excluded by either direct detection constraints, $b \rightarrow s \gamma$ constraints, constraints on the invisible decays of the Higgs, dwarf spheroidal galaxy constraints, or mass constraints on the triplet and fiveplet. Anything above the blue line is excluded.

\subsubsection{Higgs Signal Strengths}

It is convenient in this model to look at the signal strengths slightly differently. The $\kappa$ 's are defined by

$$
\kappa_{f}=\frac{g_{h f \bar{f}}}{g_{h f \bar{f}}^{S M}}
$$

and

$$
\kappa_{V}=\frac{g_{h V V}}{g_{h V V}^{S M}}
$$

as ratios of the coupling of $\mathrm{h}$ to fermions and to vector bosons. The GM model dictates that $\kappa_{f}$ is the same for all $f$, and similarly for $\kappa_{V}$ for all $V$. Rewriting the 
signal strengths in terms of these parameters, there are five different possibilities:

$$
\mu(A B \rightarrow h \rightarrow C D)=\frac{\Gamma_{T o t}^{S M}(h)}{\Gamma_{T o t}(h)}\left\{\begin{array}{l}
\kappa_{f}^{4} \\
\kappa_{V}^{4} \\
\kappa_{f}^{2} \kappa_{V}^{2} \\
\kappa_{f}^{2} \frac{\Gamma(h \rightarrow \gamma \gamma)}{\Gamma^{S M}(h \rightarrow \gamma \gamma)} \\
\kappa_{V}^{2} \frac{\Gamma(h \rightarrow \gamma \gamma)}{\Gamma^{S M}(h \rightarrow \gamma \gamma)}
\end{array}\right.
$$

where (1) occurs if the production mode is gluon gluon fusion or top associated production and the final state are fermions or two gluons, (2) occurs if the production mode is vector boson fusion or vector boson associated production and the Higgs decays to $W W^{*}$ or $Z Z^{*}$, (3) occurs if you mix cases (1) and (2), (4) occurs if you have the production modes of (1) and a final state of two photons and (5) occurs if you have the production modes of (2) and a final state of two photons.

We look at the ATLAS/CMS results using the mechanisms of the form [30]

$$
\frac{\sigma(g g \rightarrow H \rightarrow Z Z)}{\sigma(g g \rightarrow H \rightarrow Z Z)_{S M}}=\kappa_{f}^{2} \kappa_{V}^{2} \frac{\Gamma_{h}^{S M}}{\Gamma_{h}} .
$$

A chi-squared fit with the condition $\left|\Delta \chi^{2}\right|<4$ results in a parameter space for $\kappa_{f}$ and $\kappa_{V}$ shown in Figure 5.8

The parameters $\kappa_{f}$ and $\kappa_{V}$ in general do not deviate far from their standard model values $\left(\kappa_{f}=\kappa_{V}=1\right)$. The red points excluded have high enough values to contribute to high $\kappa$ values near the Higgs poles, therefore small couplings are needed to pass the relic abundance constraint.

\subsection{Summary}

The Georgi-Machacek model is extended by singlet scalar DM. After applying all constraints, singlet scalar DM masses are excluded below about $50 \mathrm{GeV}$. The tightest constraints on this model are the observed dark matter relic density and direct detection limits. There does exist, above the Higgs pole, a substantial region of parameter space yielding the correct dark matter relic density that can escape the near-future direct detection experiments DEAP and XENON 1T for dark matter 


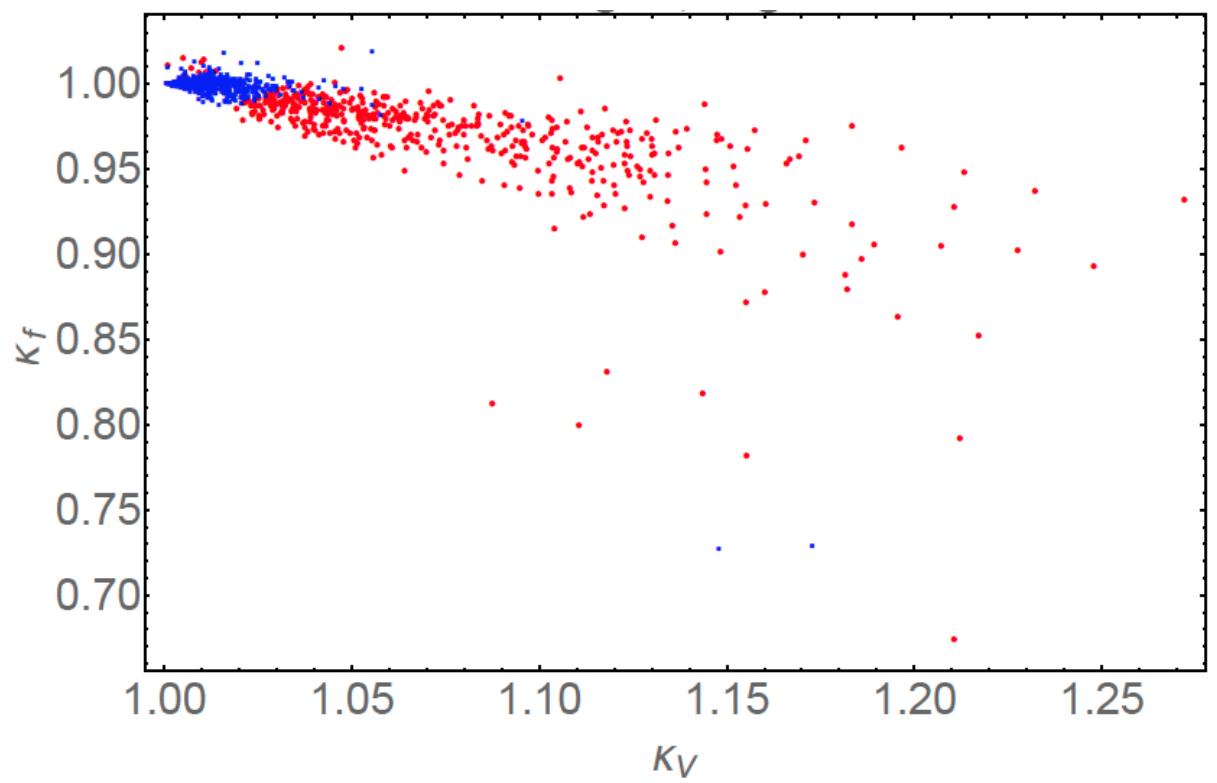

Figure 5.8: Parameter space of $\kappa_{f}$ and $\kappa_{V}$. The blue points are allowed by all previously imposed constraints.

masses as low as $125 \mathrm{GeV}$. 


\section{Chapter 6}

\section{Self-Interacting Dark Matter}

\subsection{Background and Motivation}

Recently, a study of the galaxy cluster Abell 3827 revealed a spacial offset of the dark matter halo from at least one of its central galaxies [42]. This cluster is unique in that the remnants of four eliptical galaxies are within the core radius of approximately $10 \mathrm{kpc}$. Using both Hubble Space Telescope (HST) imaging (see Figure 6.1) and integral field spectroscopy of the gravitationally lensed system through the core, the distribution of associated dark matter can be profiled. The dark matter halo in this galaxy cluster was found to be spatially offset by $\Delta=1.62_{-0.49}^{+0.47} \mathrm{kpc}$.

This offset could possibly be described by dark matter self interaction. In a highmomentum scenario, we cannot assume that the halo effects can be described by a drag force akin to classical physics. Most of the dark matter particles within the halo will not interact, but those that do will have momentum away from the core, separating from the halo. On a small time scale these will become observationally separable from the dark matter halo.

Reference [43] finds that the offset, although sensitive to the definition of the centroids of the galaxy and halo, gives an approximate cross section

$$
\frac{\sigma_{D M}}{m} \sim 1.5 \mathrm{~cm}^{2} \mathrm{~g}^{-1}
$$

In the case of contact interactions, this result is in tension with previously cited values from other astronomical systems, which give an upper bound of [44]

$$
\frac{\sigma_{D M}}{m} \leq 1 \mathrm{~cm}^{2} \mathrm{~g}^{-1}
$$




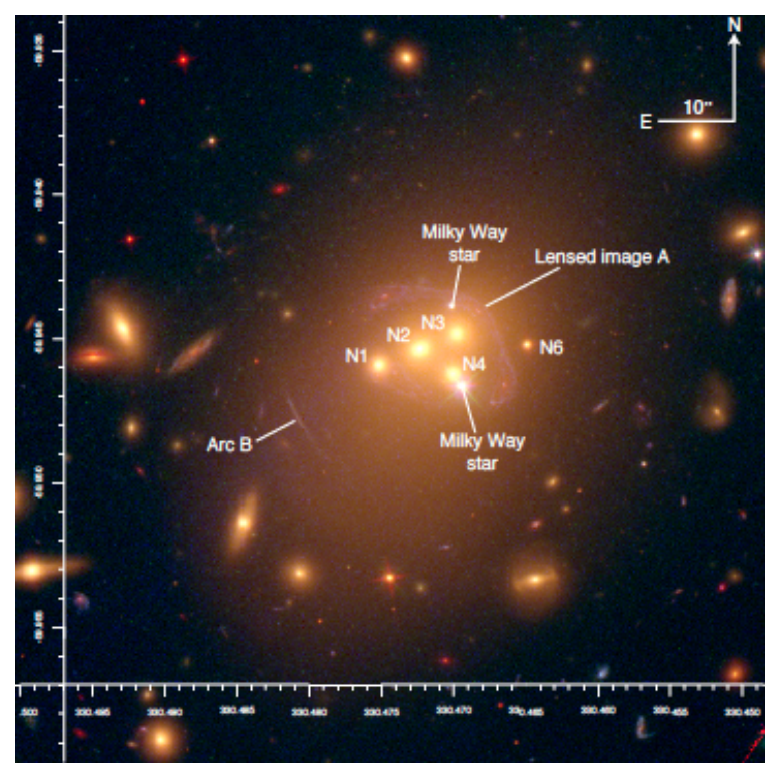

Figure 6.1: Abell 3827 HST image from Reference [42], showing the F160W (red), F160W (green) and F336W (blue) optical bands on a logarithmic colour scale. Labels N1 through N4 are the four bright central galaxies, while N5 is a star.

Both of these values will be used to constrain and thus give a range for the estimated self-interaction cross section.

The extension of the SM by a singlet scalar dark matter candidate, S, is considered, which is stable by imposing a $\mathrm{Z}_{2}$ symmetry. This couples to the rest of the SM through a "Higgs portal" operator of the form $\operatorname{SS}^{\dagger} \Phi$, where $\Phi$ is the $\mathrm{SU}(2)_{L}$ doublet SM Higgs field.

\subsection{Minimal Singlet Extension of the Standard Model}

The SM plus a real singlet scalar has the scalar potential

$$
V=-\mu^{2} \Phi^{\dagger} \Phi+\frac{\mu_{S}^{2}}{2} S S+\lambda_{h}\left(\Phi^{\dagger} \Phi\right)^{2}+\lambda_{p} S S \Phi^{\dagger} \Phi+\lambda_{S} S^{4}
$$

where

$$
\lambda_{h}=\frac{m_{h}^{2}}{2 v^{2}} \simeq 0.129
$$


and

$$
\mu^{2}=\lambda_{h} v^{2} \simeq(88.4 \mathrm{GeV})^{2} .
$$

To ensure the stability of the singlet (i.e. it does not acquire a vev), it is required that

$$
m_{S}^{2}=\mu_{S}^{2}+\lambda_{p} v^{2}>0
$$

where $m_{S}$ is the physical mass of the singlet scalar in the electroweak-breaking vacuum. It is necessary that $\mu_{S}^{2}>0$ to ensure that the potential does not develop a second minimum. To ensure boundedness from below, the conditions $\lambda_{S}>0$ and $\lambda_{p}>-2 \sqrt{\lambda_{h} \lambda_{S}}$ must also hold.

The relevant Feynman rules for the singlet vertices can be found in Equation 6.7, and the Feynman diagrams for the processes in Figure 6.2.

$$
\begin{array}{r}
S S S S:-24 i \lambda_{S} \\
S S h:-2 i \lambda_{p} v .
\end{array}
$$

There will be three DM scenarios considered: that of high mass, low mass, and mass near the Higgs resonance. Note that we will compare to the measured values in particle physics units, or, rewriting Equations 6.1 and 6.2,

$$
\sigma_{S M} / m \approx(4.7-7.0) \times 10^{3} \mathrm{GeV}^{-3} .
$$

\subsubsection{Heavy Dark Matter, $\mathbf{m}_{S} \gg \mathbf{m}_{H}$}

At a high dark matter mass, the momentum of the internal Higgs is suppressed by momentum factors, so the four-point diagram dominates. Therefore the scattering cross section per unit of dark matter mass is given by

$$
\frac{\sigma_{D M}}{m_{S}} \simeq \frac{9 \lambda_{S}^{2}}{2 \pi m_{S}^{3}}
$$

However, perturbative unitary sets an upper bound on $\lambda_{S}$ of $\frac{2 \pi}{3}$, due to identical particles in the final state and requirements of the zeroth partial wave amplitude. This results in a self interaction cross section that is inconsistent with the measured 

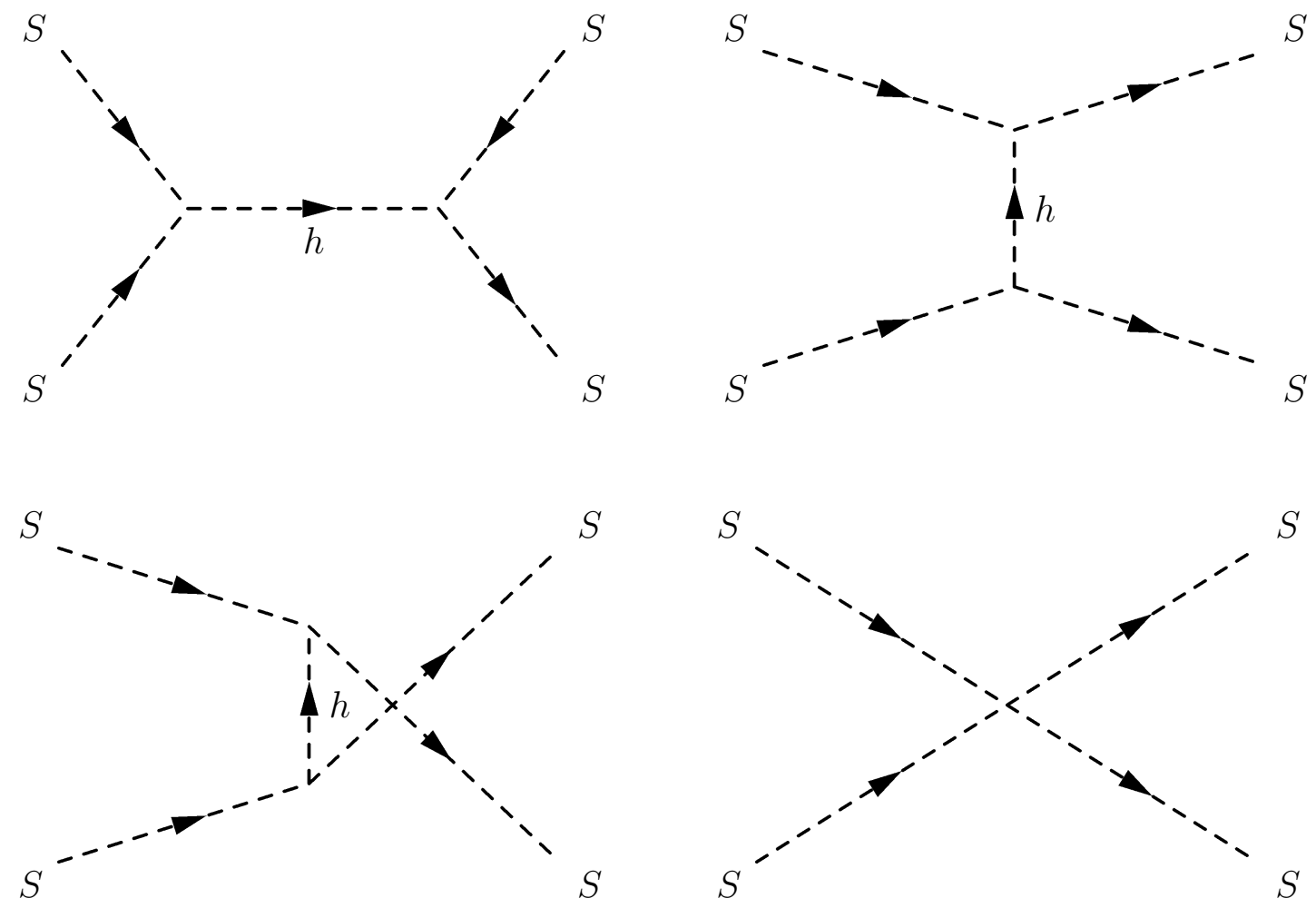

Figure 6.2: Feynman diagrams for the interaction $S S \rightarrow S S$

value in Equation 6.8. In fact, reproducing the measured dark matter interaction cross section requires low-mass dark matter, or $m_{S} \leq 0.1 \mathrm{GeV}$, thus ruling out the heavy DM scenario.

\subsubsection{Dark matter near the Higgs resonance}

At a mass near the Higgs resonance $\left(m_{S} \simeq m_{h} / 2\right)$, the s-channel exchange diagram dominates as in Figure 6.3. In this case, the cross section per unit mass is

$$
\begin{aligned}
\frac{\sigma_{D M}}{m_{S}} & \simeq \frac{g_{S S h}^{4}}{128 \pi m_{S}^{3}\left(4 m_{S}^{2}-m_{h}^{2}\right)^{2}} \\
& \simeq \frac{v^{4} \lambda_{p}^{4}}{16 \pi m_{h}^{5}\left(m_{S}-m_{h} / 2\right)^{2}}
\end{aligned}
$$


where $i g_{S S h}$ is the hSS Feynman coupling, and $m_{S} \approx m_{h} / 2$. An additional constraint comes from perturbative unitary, yielding $\left|g_{S S h}\right|<8 \pi v$. This requires severe tuning of $\mathrm{m}_{S}$, such that $\left|m_{S}-m_{h} / 2\right| \leq 0.11 \mathrm{GeV}$.
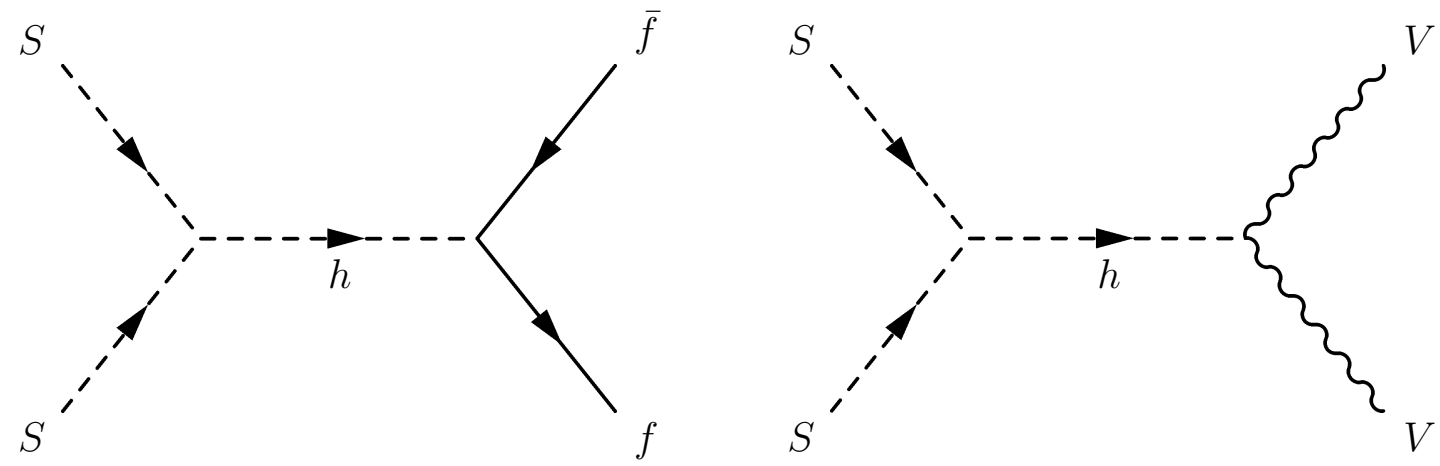

Figure 6.3: Feynman diagrams for the annihilation of DM into SM particles in the real singlet scalar extension of the SM.

We can try to reconcile this scenario using the observed dark matter relic density. The value of the corresponding annihilation cross section is

$$
\sigma v_{r e l} \approx 2.2 \times 10^{-26} \mathrm{~cm}^{3} \mathrm{~s}^{-1} \approx 1.9 \times 10^{-9} \mathrm{GeV}^{-2}
$$

where $v_{\text {rel }}$ is the relative velocity between the two dark matter particles in the centre of momentum frame. The annihilation cross section can be written as

$$
\sigma v_{r e l}=\frac{2 g_{S S h}^{2} \Gamma_{t o t}^{h_{S M}}}{E_{c m}\left(E_{c m}^{2}-m_{h}^{2}\right)^{2}}
$$

where $E_{c m}=2 m_{S}+\frac{1}{4} m_{S} v_{r e l}^{2}$ is the centre of mass energy and $\Gamma_{t o t}^{h_{S M}}$ is the total width of the SM Higgs (4.1 MeV) [45]. If the singlet scalar is heavier than half the Higgs mass, the thermal motion pushes the annihilation process far from resonance. Integrating the annihilation cross section over the Boltzmann distribution under the assumption $\mathrm{T}=m_{S} / 20$ gives

$$
\left\langle\sigma v_{r e l}\right\rangle \approx 0.03 \mathrm{GeV}^{-2}
$$

This is many orders of magnitude greater than the required cross section from Equation 6.12 , so that the relic abundance would be too small to account for the 
observed dark matter. If the singlet scalar is lighter than half the Higgs mass, the cross section becomes even larger and forces the relic abundance smaller, due to the large hSS coupling and the tuning of the singlet mass.

\subsubsection{Low-mass dark matter, $\mathrm{m}_{S} \ll \mathrm{m}_{h}$}

At low mass $\left(m_{S} \ll m_{h}\right)$, all four of the Feynman diagrams in Figure 6.2 will contribute to dark matter self annihilation. At low energies the Higgs boson can be integrated out, which yields an effective 4-point coupling with Feynman rule $-24 \mathrm{i} \lambda_{e f f}$, where

$$
\lambda_{e f f}=\lambda_{S}-\frac{g_{S S h}^{2}}{8 m_{h}^{2}} .
$$

Again, bounds from perturbative unitarity must be applied, giving the upper bound $\left|\lambda_{e f f}\right|<2 \pi / 3$. The DM scattering cross section as in Equation 6.9 becomes

$$
\frac{\sigma_{D M}}{m_{S}} \approx \frac{9 \lambda_{e f f}^{2}}{2 \pi m_{S}^{3}}
$$

These results are consistent with Reference [43] if the dark matter mass is very light, ie below about $0.1 \mathrm{GeV}$, as shown in Figure 6.4.

\subsubsection{Phenomenology}

\section{Invisible Higgs Decays}

In the low mass range $m_{S} \lesssim 0.1 \mathrm{GeV}$, the parameter $\lambda_{p}$ is constrained by a lack of observation of invisible Higgs decays. The expression for the invisible partial width is

$$
\Gamma(h \rightarrow S S)=\frac{g_{S S h}^{2}}{32 \pi m_{h}} \sqrt{1-\frac{4 m_{S}^{2}}{m_{h}^{2}}} \simeq \frac{g_{S S h}^{2}}{32 \pi m_{h}}
$$

where the last approximation holds for $m_{S}<<m_{h}$. Again, the strongest constraint on the branching ratio (Equation 3.3) is $\mathrm{BR}(\mathrm{h} \rightarrow$ invis.) $<0.28$, coming from ATLAS bounds on vector boson fusion with a $125 \mathrm{GeV}$ Higgs and a SM production cross section [19]. Therefore the upper bound on $g_{S S h}$ is 


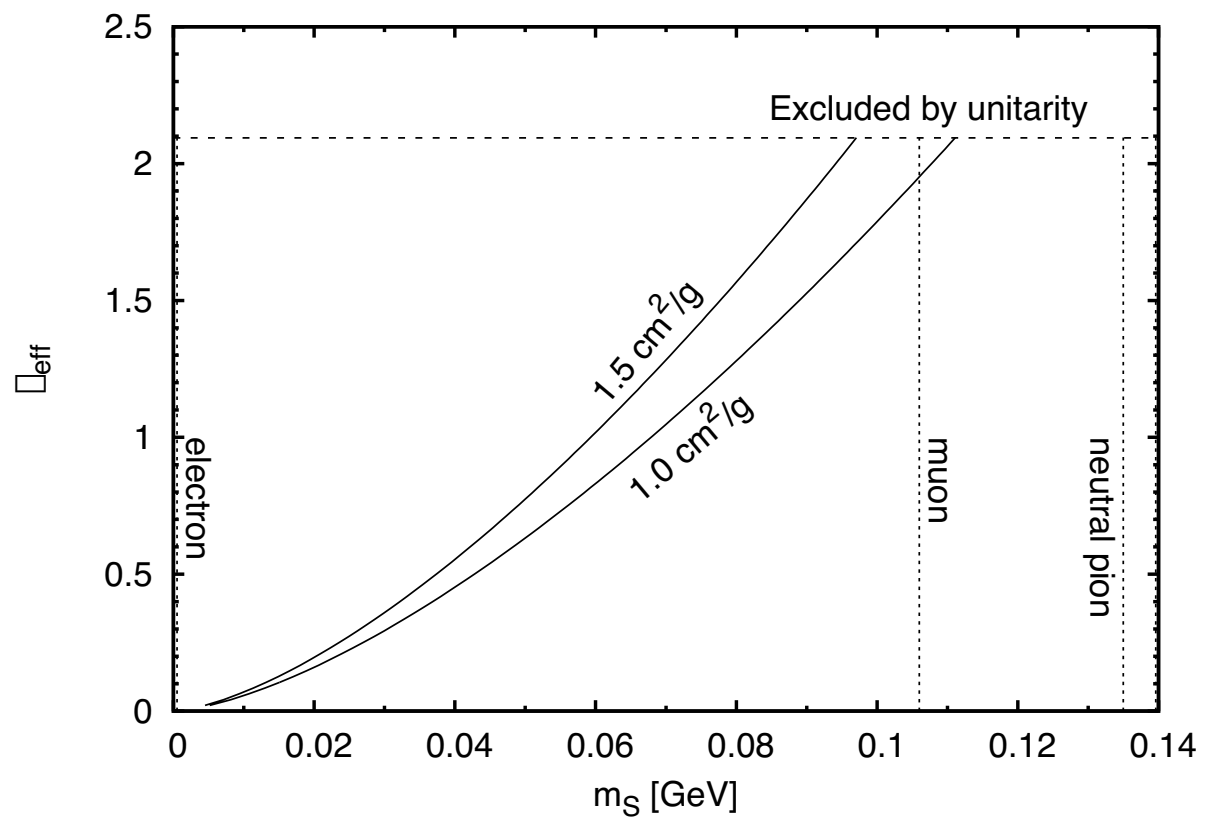

Figure 6.4: The allowed region for $m_{S}$ and $\left|\lambda_{e f f}\right|$ to obtain the desired range in Equation 6.8. This includes the constraints from perturbative unitarity and thresholds for DM annihilation to electrons, muons, and neutral pions.

$$
g_{S S h}^{2}<21 \mathrm{GeV}^{2} \quad \text { or } \quad\left|\lambda_{p}\right|<9.3 \times 10^{-3},
$$

which is strong when compared to the unitary constraint $\left|\lambda_{p}\right|<4 \pi$.

An upper bound on $g_{S S h}$ can also be found from the measured signal strengths of the Higgs boson. In the model SM + scalar singlet, all signal strengths are multiplied by the factor

$$
\mu_{i}=\frac{\Gamma_{t o t}^{h s m}}{\Gamma_{t o t}^{h s m}+\Gamma(h \rightarrow S S)} .
$$

ATLAS and CMS measure an overall signal strength of

$$
\begin{array}{ll}
\mu=1.18_{-0.14}^{+0.15} & (\text { ATLAS }) \\
\mu=1.00_{-0.13}^{+0.14} & (\text { CMS })
\end{array}
$$

However, there is an additional constraint on this model in particular, that $\mu \leq 1$, so the $\Delta \chi^{2}=4$ lower bounds are 


$$
\begin{aligned}
& \mu \geq 0.85 \quad \text { (ATLAS) } \\
& \mu \geq 0.74 \quad \text { (CMS). }
\end{aligned}
$$

These lead to upper bounds on $g_{s s h}^{2}$ :

$$
\begin{aligned}
& g_{S S h}^{2} \leq 9.1 \mathrm{GeV}^{2} \quad \text { or } \quad\left|\lambda_{p}\right| \leq 6.1 \times 10^{-3} \quad \text { (ATLAS) }, \\
& g_{S S h}^{2} \leq 18 \mathrm{GeV}^{2} \text { or }\left|\lambda_{p}\right| \leq 8.6 \times 10^{-3} \quad \text { (CMS). }
\end{aligned}
$$

\section{Relic Density}

For small $m_{S} \sim 0.1$, the annihilation cross section must be [46]

$$
\sigma v_{\text {rel }} \simeq 4.8 \times 10^{-26} \mathrm{~cm}^{3} \mathrm{~s}^{-1} \simeq 4.1 \times 10^{-9} \mathrm{GeV}^{-2}
$$

At this low mass, the dark matter annihilation is predominantly s-channel, as in Figure 6.3. This can be expressed in terms of the total width of the SM Higgs if the dark matter were half the Higgs mass:

$$
\sigma v_{r e l} \simeq \frac{4 v^{2} \lambda_{p}^{2}}{m_{h}^{4}} \frac{\Gamma_{t o t}^{h_{S M}}\left(m_{h}=2 m_{S}\right)}{m_{S}}
$$

As shown in Figure 6.4, the highest annihilation cross section is found at the high end of the singlet mass range, when annihilations into muons are kinematically accessible, and $\Gamma_{t o t}^{h_{S M}}\left(m_{h}=2 m_{S}\right)$ can be approximated by the width into muons:

$$
\Gamma\left(h^{*} \rightarrow \mu \mu\right)=\frac{1}{8 \pi} \frac{m_{\mu}^{2}}{v^{2}}\left(2 m_{S}\right)\left(1-\frac{m_{\mu}^{2}}{m_{S}^{2}}\right)
$$

which, substituting into Equation 6.24, gives

$$
\sigma v_{r e l} \simeq\left(1.5 \times 10^{-11} \mathrm{GeV}^{-2}\right) \lambda_{p}^{2}\left(1-\frac{m_{\mu}^{2}}{m_{S}^{2}}\right)^{\frac{3}{2}}
$$

A large enough value of the annihilation cross section cannot be obtained from constraints on perturbative unitarity alone, not to mention constraints on the muon mass and $\left|\lambda_{p}\right|$ from LHC Higgs data. In fact, the constraint on $\left|\lambda_{p}\right|$ from invisible Higgs decays and signal strengths results in the annihilation cross section to be six orders 
of magnitude too small to yield the correct relic abundance. Furthermore, the low mass scenario with $S S \rightarrow \mu \mu$ is ruled out by relic abundance constraints.

\section{Thermal Freeze-In}

The correct dark matter relic abundance for thermal freezeout cannot be obtained by this model, for the cross section to SM particles cannot be made large enough. However, there is an alternate way to obtain the relic abundance: through thermal freeze-in $[47,48]$. Here, the cross section of dark matter annihilating to SM particles is extremely small, so that thermal equilibrium is never reached. Instead, dark matter is produced through annihilations of SM particles, until the universe becomes too cool to produce dark matter, freezing in the current amount of dark matter. The annihilation of dark matter to SM particles become kinematically irrelevant.

This scenario was previously studied in references $[47,49]$. In the low mass region $m_{S} \leq 0.1 \mathrm{GeV}$, the correct dark matter relic abundance is obtained for $\left|\lambda_{p}\right| \sim(2-$ $3) \times 10^{-11}$. This is consistent with the upper bound $\left|\lambda_{p}\right|<(6.1-9.3) \times 10^{-3}$ from LHC signal strengths and invisible width branching ratio limits. The only problem is in the phenomenological sense: the dark matter becomes impossible to detect through non-gravitational interactions with SM particles.

\subsection{Two Higgs Doublet Model with Singlet DM}

The thermal freeze-out scenario is now considered for the Two Higgs doublet model $(2 \mathrm{HDM})$, again with singlet scalar dark matter. The 2HDM is characterized by a SM Higgs with mass $125 \mathrm{GeV}$, and a second CP-even scalar, $\mathrm{H}$, which could be lighter or heavier than $\mathrm{h}$.

Previously, the resonant-scattering produced a cross section far too large for DM annihilating through a Higgs portal. For this new scenario, the DM interacts resonantly through the $\mathrm{H}$, where the thermal DM annihilation cross section can be suppressed through the coupling of $\mathrm{H}$ to SM particles.

\subsubsection{Type I 2HDM}

The scalar potential of the $2 \mathrm{HDM}$ with a singlet scalar DM extension is 


$$
V=\frac{\mu_{S}^{2}}{2} S^{2}+\lambda_{S} S^{4}+\lambda_{p 1} S S \Phi_{1}^{\dagger} \Phi_{1}+\lambda_{p 2} S S \Phi_{2}^{\dagger} \Phi_{2}+V_{2 H D M} .
$$

Here, $\Phi_{1}$ and $\Phi_{2}$ are the two Higgs doublets, and $\mathrm{V}_{2 H D M}$ is the scalar potential of the 2HDM.

The class of $2 \mathrm{HDM}$ s can be divided into "types" according to symmetries and couplings. The soft breaking of the $\mathrm{Z}_{2}$ symmetry $\left(\Phi_{1} \rightarrow-\Phi_{1}\right)$ forces the righthanded fermions to only couple to one of the Higgs doublets [50]. This transformation is classified as a "Type I" 2HDM.

The vacuum expectation values are as follows:

$$
\left\langle\Phi_{1}\right\rangle=\left(\begin{array}{c}
0 \\
v_{1} / \sqrt{2}
\end{array}\right), \quad\left\langle\Phi_{2}\right\rangle=\left(\begin{array}{c}
0 \\
v_{2} / \sqrt{2}
\end{array}\right),
$$

where normalization gives $v_{1}^{2}+v_{2}^{2}=v^{2}$, and $\tan \beta=v_{2} / v_{1}$ by definition.

In terms of the real neutral components of the two doublets $\Phi_{i}^{0} \equiv \phi_{i}^{0, r} / \sqrt{2}$, the physical CP-even neutral scalars $h$ and $H$ are defined as

$$
\begin{aligned}
h & =-\sin \alpha \phi_{1}^{0, r}+\cos \alpha \phi_{2}^{0, r}, \\
H & =\cos \alpha \phi_{1}^{0, r}+\sin \alpha \phi_{2}^{0, r},
\end{aligned}
$$

where $\alpha$ is a mixing angle.

The first constraint comes from perturbative unitarity of the scattering amplitudes $S S \rightarrow \Phi_{1(2)} \Phi_{1(2)}$, which constrains the Higgs-DM couplings:

$$
\left|\lambda_{p 1}\right|<4 \pi, \quad\left|\lambda_{p 2}\right|<4 \pi
$$

The Feynman rules for the SSh and SSH vertices are $i g_{S S h}$ and $i g_{S S H}$, respectively, where

$$
\begin{array}{r}
g_{S S h}=2 v\left(\lambda_{p 1} \cos \beta \sin \alpha-\lambda_{p 2} \sin \beta \cos \alpha\right) \\
g_{S S H}=2 v\left(-\lambda_{p 1} \cos \beta \cos \alpha-\lambda_{p 2} \sin \beta \sin \alpha\right) .
\end{array}
$$

The low-energy effective $S S \rightarrow S S$ coupling for low $\mathrm{m}_{S}$ is given by 


$$
\lambda_{e f f}=\lambda_{S}-\frac{g_{S S h}^{2}}{8 m_{h}^{2}}-\frac{g_{S S H}^{2}}{8 m_{H}^{2}}
$$

The constraint from the previous section $m_{S} \lesssim 0.1 \mathrm{GeV}$ is still valid here, so we do not continue the low-mass analysis.

To avoid experimental constraints from $\mathrm{h}$ coupling measurements, we tune $\sin (\beta-$ $\alpha \simeq 1$, implying $\sin \alpha \simeq-\cos \beta$ and $\cos \alpha \simeq \sin \beta$. For $\sin (\beta-\alpha) \simeq 1$, the coupling between $\mathrm{H}$ and pairs of vector bosons is heavily suppressed, as it is proportional to $\cos (\beta-\alpha)$. In this Type-I model, fermion Yukawa couplings can be suppressed by making $\cot \beta$ small.

The $\mathrm{H}$ coupling to fermions is proportional to the SM Higgs coupling, with scaling factor

$$
\kappa_{F}^{H}=\frac{\sin \alpha}{\sin \beta} \simeq-\cot \beta,
$$

where the approximation corresponds to the $\sin (\beta-\alpha) \rightarrow 1$ limit.

If $m_{S} \approx m_{H} / 2$, dark matter scattering $S S \rightarrow S S$ is dominated by the s-channel $\mathrm{H}$ exchange diagram. The resulting cross section per unit mass is

$$
\sigma_{D M} / m_{S} \simeq \frac{g_{S S H}^{4}}{128 \pi m_{S}^{3}\left(4 m_{S}^{2}-m_{H}^{2}\right)^{2}} .
$$

Given the constraints on the vertex factors, the singlet mass must be tuned very close to $m_{H} / 2$ to obtain a cross section compatible with the halo displacement from Reference [43].

The dark matter annihilation cross section is given for annihilation through the $\mathrm{H}$ resonance by

$$
\sigma v_{r e l} \simeq \frac{2 g_{S S H}^{2} \Gamma_{t o t}^{H \rightarrow S M}}{E_{c m}\left(E_{c m}^{2}-m_{H}^{2}\right)^{2}}
$$

which is of the same form as the cross section in Equation 6.34. For the scalar singlet extension of the SM, the problem arose from the dark matter self-interaction cross section causing the annihilation cross section to be too large. Therefore $g_{S S H}$ must be large while the H producing SM particles are also suppressed.

It is assumed that $m_{H}>m_{h}$, so that $m_{S}>m_{h} / 2$ and invisible decay are kinematically forbidden. To suppress the $\mathrm{W}$ and $\mathrm{Z}$ decay channels, it is assumed that $\sin (\beta-\alpha) \rightarrow 1$, and the fermions can be suppressed taking $\cot \beta<<1$. 
The decay width of $\mathrm{H}$ to SM particles (assuming $m_{H}<2 m_{t}$ ) is

$$
\Gamma_{t o t}^{H \rightarrow S M} \simeq\left(\kappa_{f}^{H}\right)^{2}\left(\frac{m_{H}}{125 G e V}\right)\left[1-B R\left(h_{S M} \rightarrow V V\right)\right] \Gamma_{t o t}^{h S M}
$$

where

$$
B R\left(h_{S M} \rightarrow V V\right) \equiv B R\left(h_{S M} \rightarrow W W\right)+B R\left(h_{S M} \rightarrow Z Z\right) \simeq 0.24
$$

and

$$
\Gamma_{t o t}^{H \rightarrow S M} \propto\left(\kappa_{f}^{H}\right)^{2} \simeq \cot ^{2} \beta
$$

Comparing this expression with the annihilation cross section in Equation 6.34, the parameter combination $\left(\kappa_{f}^{H}\right)^{2} / g_{S S H}^{2}$ must be extremely suppressed. However, taking into account the approximations, the SSH coupling is

$$
g_{S S H} \simeq 2 v \sin \beta \cos \beta\left(-\lambda_{p 1}+\lambda_{p 2}\right),
$$

which is proportional to $\cos \beta$, and is therefore not suppressed by $\cot \beta<<1$. Once the upper bounds from perturbative unitarity are imposed $\left(\left|g_{S S h}\right|<8 \pi v\right)$, the situation is no better than the previous SM+singlet scenario.

\subsubsection{Type II 2HDM}

The above problems could be avoided if the scalar potential contained the term $\left(S S \Phi_{1}^{\dagger} \Phi_{2}+\right.$ h.c.), breaking the $Z_{2}$ symmetry. This is referred to as the Type II 2HDM, and allows fermions to couples to both Higgs. We add an additional term to the potential in Equation 6.3:

$$
V \supset\left(\lambda_{p 3} S S \Phi_{1}^{\dagger} \Phi_{2}+\text { h.c. }\right)
$$

where $\lambda_{p 3}$ is real to avoid CP violation. The Feynman rule parameters become

$$
\begin{array}{r}
g_{S S h}=2 v\left[\lambda_{p 1} \cos \beta \sin \alpha-\lambda_{p 2} \sin \beta \cos \alpha-\lambda_{p 3}(\cos \beta \cos \alpha-\sin \beta \sin \alpha)\right] \\
g_{S S H}=2 v\left[-\lambda_{p 1} \cos \beta \cos \alpha-\lambda_{p 2} \sin \beta \sin \alpha-\lambda_{p 3}(\cos \beta \sin \alpha+\sin \beta \cos \alpha)\right]
\end{array}
$$

Tuning $\sin (\beta-\alpha) \simeq 1$ to make the h couplings SM-like reduces these to 


$$
\begin{array}{r}
g_{S S h} \simeq 2 v\left[\lambda_{p 1} \cos ^{2} \beta-\lambda_{p 2} \sin ^{2} \beta-2 \lambda_{p 3} \sin \beta \cos \beta\right] \\
g_{S S H} \simeq 2 v\left[\left(-\lambda_{p 1}+\lambda_{p 2}\right) \sin \beta \cos \beta-\lambda_{p 3}\left(\sin ^{2} \beta-\cos ^{2} \beta\right)\right] .
\end{array}
$$

Now, a supression of the $\left(\kappa_{f}^{H}\right)^{2}$ factor in Equation 6.35 need not be counteracted by a suppression of $g_{S S H}^{2}$. Choosing $\kappa_{f}^{H} \sim 10^{-4}$ gives an annihilation cross section in the right range for thermal freezeout.

\subsection{Summary}

The apparent observation of dark matter self-interactions in the cluster Abell 3827 are used to constrain models of singlet scalar dark matter. Thermal freezeout in the simple case of the SM with a singlet scalar DM candidate produces an annihilation cross section six orders of magnitude too small to account for the observed relic abundance. The most natural solution for this deficiency is very light dark matter (below about $0.1 \mathrm{GeV}$ ), whose relic abundance is set by freeze-in through extraordinarily weak interactions with the Higgs doublet(s) through a quartic coupling of order $10^{-11}$. Two-Higgs doublet models are examined in hopes of a better result. The solution is, however, quite fine tuned: the singlet mass must be tuned to a value very close (of order a part per mille) to half the mass of the second CP-even neutral Higgs boson. 


\section{Chapter 7}

\section{Summary}

The question of dark matter continues to be one to unite experiment and theory in the search for a solution. This thesis considers models of BSM physics that incorporate a singlet scalar DM candidate, and uses theoretical and experimental constraints to constrain model parameters.

In Section 4 the phenomenology of a model is studied that treats the physical Higgs boson of the SM as a dilaton, the pseudo-Goldstone boson of spontaneous conformal symmetry breaking, and additionally introduces a scalar singlet dark matter candidate. Three of the six parameters of the model were fitted to the properties of the $125 \mathrm{GeV}$ Higgs boson properties. Constraints from DM experiments were then implemented. It was found that the magnitudes of $c_{g g}$ and $c_{\gamma \gamma}$ are constrained to be close to the standard model values. It was also found that masses less than $\sim 50 \mathrm{GeV}$ are excluded by the allowed values of DM relic abundance. When direct detection limits by the XENON and LUX experiments are imposed, only a small mass region remains assuming a SM-like dilaton self coupling. When one assumes the self coupling is more like a strongly interacting composite theory a second region survives in the mass region of $125-300 \mathrm{GeV}$. The higher mass region will be probed in the near future by the DEAP DM search experiment. The limited region of parameter space allowed by direct detection experiments is enhanced when one allows for a negative value of $c_{g g}$. Furthermore, future precision measurements on the parameters governing the Higgs potential can indicate whether the scalar observed is the scalar responsible for the Brout-Englert-Higgs mechanism of electroweak symmetry breaking or a dilaton. If these measurements deviate from the SM expectation, they reveal more about the conformal field theory for energies $f \gtrsim 246 \mathrm{GeV}$ and the mechanism responsible for a light dilaton, given that its natural mass will be on the order of $4 \pi f$. 
In Section 5 the Georgi-Machacek model with a SSDM candidate is studied. The GM model extends the Standard Model Higgs sector with the addition of isospintriplet scalar fields in such a way as to preserve the custodial symmetry. The singlet scalar is required to account for the full relic abundance of dark matter in the universe. The combination of the observed dark matter relic density and direct detection constraints exclude singlet scalar masses below about $50 \mathrm{GeV}$. It is also found that, in the parameter space above the Higgs pole, the correct dark matter relic density can escape the near-future direct detection experiments DEAP and XENON 1T for dark matter masses as low as $125 \mathrm{GeV}$. This is in contrast to the singlet scalar dark matter extension of the Standard Model, for which these future experiments are expected to exclude dark matter masses above the Higgs pole up to the multi-TeV range.

Finally, in Section 6 the evidence for dark matter self-interactions based on the observation of a spatial offset between the dark matter halo and the stars in a galaxy in the cluster Abell 3827 is considered. Interpreting the offset as due to dark matter self-interactions leads to a cross section measurement of $\mathrm{DM} / \mathrm{m}=(1-1.5) \mathrm{cm}^{2} \mathrm{~g}^{-1}$, where $\mathrm{m}$ is the mass of the dark matter particle. This observation is used to constrain singlet scalar dark matter extensions of the standard model and two-Higgs-doublet models. We show that the most natural scenario in this class of models is very light dark matter, below about $0.1 \mathrm{GeV}$, whose relic abundance is set by thermal freeze-in. It is also shown that the dark matter abundance can be established through the usual thermal freeze-out mechanism in the singlet scalar extension of the Yukawa-aligned two-Higgs-doublet model, but that it requires severe fine tuning of the singlet scalar mass.

Future work may include reconsidering the Higgs-like dilaton with other options for dark matter (i.e. composite DM). There are also other existing models that could be put through the set of constraints outlined in this thesis to study the addition of a dark matter candidate. 


\section{References}

[1] R. Campbell, S. Godfrey, H. E. Logan, A. D. Peterson, and A. Poulin, "Implications of the observation of dark matter self-interactions for singlet scalar dark matter," Phys. Rev., vol. D92, no. 055031, 2015.

[2] R. Campbell, S. Godfrey, and A. de la Puente, "The dilaton-like higgs boson with scalar singlet dark matter," arXiv:hep-ph/1607.02158, 2016.

[3] R. Campbell, S. Godfrey, H. E. Logan, and A. Poulin, "The real singlet scalar dark matter extension of the georgi-machacek model," In progress, 2016.

[4] LIGO, "Gravitational waves detected 100 years after einstein's prediction," February 2016.

[5] T. C. Collaboration, "Observation of a new boson at a mass of 125 gev with the cms experiment at the lhc," Phys. Lett. B, vol. 716, no. 30, 2012.

[6] T. A. collaboration, "Search for resonances decaying to photon pairs in $3.2 \mathrm{fb}^{-1}$ of $p p$ collisions at $\sqrt{s}=13$ tev with the atlas detector," ATLAS-CONF-2015-081, 2015 .

[7] D. Griffiths, Introduction to elementary particles. Wiley-VCH, 2008.

[8] T. L. Collaboration, "Observation of $\mathrm{j} / \psi$ resonances consistent with pentaquark states in $\lambda_{b}^{0} \rightarrow j / \psi k^{-} p$ decays," Phys. Rev. Lett., vol. 115, no. 072001, 2015.

[9] M. Peskin and D. Schroeder, An introduction to quantum field theory. Westview Press, 1995.

[10] K. Nguyen, "The higgs mechanism," uni-muenchen.de, 2009.

[11] S. Dawson, "Introduction to electroweak symmetry breaking," in Proceedings, Summer School in High-energy physics and cosmology, pp. 1-83, 1998.

[12] S. Dawson, "Electroweak symmetry breaking circa 2005," Int. J. Mod. Phys. A., vol. 21, pp. 1629-1641, 2006.

[13] G. Giudice, "Theoretical predictions for collider searches," Int. J. Mod. Phys., vol. A19, pp. 835-850, 2004.

[14] E. Fischer, "The properties of dark matter," ArXiv e-prints, vol. 1104.2525, 2011. 
[15] F. Zwicky, "On the masses of nebulae and of clusters of nebulae," Astrophysicial Journal, vol. 86, p. 217, 1937.

[16] G. B. Gelmini, "Tasi 2014 lectures: The hunt for dark matter," arXiv:hepph/1502.01320, 2015.

[17] V. C. Rubin and W. K. J. Ford, "Rotation of the andromeda nebula from a spectroscopic survey of emission regions," Astrophysical Journal, vol. 159, p. 379, 1970.

[18] G. Aad et al., "Measurements of the higgs boson production and decay rates and constraints on its couplings from a combined atlas and cms analysis of the lhe $p p$ collision data at $\sqrt{s}=7$ and 8 tev," arXiv:hep-ph/1606.02266, 2016.

[19] G. Aad et al., "Constraints on new phenomena via higgs boson couplings and invisible decays with the atlas detector," JHEP, vol. 11, no. 10.1007, p. 206, 2015.

[20] E. W. Kolb and M. S. Turner, The Early Universe. Addison-Wesley Publishing Company, 1989.

[21] P. A. R. Ade et al., "Planck 2013 results. xvi. cosmological parameters," Astron. Astrophys., vol. 571, p. A16, 2014.

[22] T. Marrodan Undagoitia and L. Rauch, "Dark matter direct-detection experiments," J. Phys, vol. G43, no. 1, p. 013001, 2016.

[23] J. Angle et al., "A search for light dark matter in xenon10 data," Phys. Rev. Lett., vol. 107, p. 051301, 2011.

[24] D. S. Akerib et al., "First results from the lux dark matter experiment at the sanford underground research facility," Phys. Rev. Lett., vol. 112, p. 091303, 2014.

[25] "http://www.xenon1t.org," 2016.

[26] "http://luxdarkmatter.org," 2016.

[27] P. A. Amaudruz et al., "Deap-3600 dark matter search," Nucl. Part. Phys. Proc., vol. 273-275, pp. 340-346, 2016.

[28] F. S. Queiroz, "Dark matter overview: Collider, direct and indirect detection searches," in 1605.08788, 2016.

[29] B. Bellazzini, C. Csaki, J. Hubisz, J. Serra, and J. Terning, "A higgslike dilaton," Eur. Phys. J, vol. C73, no. 2, p. 2333, 2013.

[30] G. Aad et al., "Measurements of the higgs boson production and decay rates and coupling strengths using pp collision data at $\sqrt{s}=7$ and 8 tev in the atlas experiment," Eur. Phys. J, vol. C76, no. 1, p. 6, 2016. 
[31] M. Ciuchini, E. Franco, S. Mishima, and L. Silverstrini, "Electroweak precision observables, new physics, and the nature of 126 gev higgs boson," JHEP, vol. 08, p. 106, 2013.

[32] V. Barger, M. Ishida, and W. Y. Keung, "Total width of 125 gev higgs boson," Phys. Rev. Lett., vol. 85, no. 055001, 2012.

[33] CMSCollaboration, "Constraints on the higgs boson width from off-shell production and decay to z-boson pairs," Phys. Lett. B, vol. 736, no. 64, 2014.

[34] G. Belanger, F. Boudjema, A. Pukhoc, and A. Semenov, "Dark matter direct detection rate in a generic model with micromegas 2.2," Comput. Phys. Commun., vol. 180, pp. 747-767, 2009.

[35] J. M. Cline, K. Kainulainen, P. Scott, and C. Weniger, "Update on scalar singlet dark matter," Phys. Rev., vol. D88, p. 055025, 2013.

[36] "dmtools.brown.edu," 2016.

[37] H. Georgi and M. Machacek, "Doubly charged higgs bosons," Nucl. Phys. B, vol. 262, no. 3, pp. 463-477, 1985.

[38] H. E. Logan and V. Rentala, "All the generalized georgi-machacek models," Phys. Rev., vol. D92, no. 7, p. 075011, 2015.

[39] K. Hartling, K. Kumar, and H. E. Logan, "The decoupling limit in the georgimachacek model," Phys. Rev., vol. D90, no. 015007, 2014.

[40] K. Hartling, K. Kumar, and H. E. Logan, "Gmcalc: a calculator for the georgimachacek model," arXiv:hep-ph/1412.7387, 2014.

[41] J. Beringer and et al, "Status of higgs boson physics," Phys. Rev., vol. D86, p. 010001, 2014.

[42] R. Massey et al., "The behaviour of dark matter associated with four bright cluster galaxies in the $10 \mathrm{kpc}$ core of abell 3827," Mon. Not. Roy. Astron. Soc., vol. 449, no. 4, pp. 3393-3406, 2015.

[43] F. Kahlhoefer, K. Schmidt-Hoberg, J. Kummer, and S. Sarkar, "On the interpretation of dark amtter self-interactions in abell 3827," Mon. Not. Roy. Astron. Soc., vol. 452, no. 1, pp. L54-L58, 2015.

[44] M. Markevitch, A. H. Gonzalez, D. Clowe, A. Vikhlinin, L. David, W. Forman, C. Jones, S. Murray, and W. Tucker, "Direct constraints on the dark matter selfinteraction cross-section from the merging galaxy cluster 1e0657-56," Astrophys. J., vol. 606, pp. 819-824, 2004.

[45] J. R. Anderson et al., "Handbook of lhe higgs cross sections: 3. higgs properties," arXiv:hep-ph/130\%.1347, 2013.

[46] G. Steigman, B. Dasgupta, and J. F. Beacom, "Precise relic wimp abundance and its impact on searches for dark matter annihilation," Phys. Rev., vol. D86, p. $023506,2012$. 
[47] J. McDonald, "Thermally generated gauge singlet scalars as self interacting dark matter," Phys. Rev. Lett., vol. 88, p. 091304, 2002.

[48] L. J. Hall, K. Jedamzik, J. March-Russell, and S. M. West, "Freeze-in production of fimp dark matter," JHEP, vol. 03, p. 080, 2010.

[49] C. E. Yaguna, "The singlet scalar as fimp dark matter," JHEP, vol. 08, p. 060, 2011.

[50] G. C. Branco, P. M. Ferreira, L. Lavoura, M. N. Rebelo, M. Sher, and J. P. Silva, "Theory and phenomenology of two-higgs-doublet models," Phys. Rept., vol. 516, pp. 1-102, 2012. 


\section{Appendix A}

\section{Feynman Rules}

\section{A.1 Higgs-like Dilaton with Scalar Singlet Dark Matter}

The Feynman rules in this section are written in terms of the vertex -ig $\operatorname{sbc}_{a b}$.

Feynman rules for couplings to $S$

The Feynman rules for the coupling of the dilaton to $S$ are

$$
\begin{array}{r}
g_{S S \sigma}=\frac{-2 i \epsilon m_{S}^{2}}{v^{2}} \\
g_{S S \sigma \sigma}=\frac{-4 i \epsilon^{2} m_{S}^{2}}{v^{2}} .
\end{array}
$$

Feynman rules for couplings to $\sigma$

The Feynman rules for the dilaton coupling to SM particles are as follows: 


$$
\begin{aligned}
g_{\sigma \gamma \gamma} & =\frac{i c_{\gamma \gamma} \epsilon p_{1}^{\mu_{2}} p_{2}^{\mu_{1}}}{2 \pi \alpha_{E M} v}-\frac{i c_{\gamma \gamma} \epsilon g_{\mu_{1} \mu_{2}} p_{1} \cdot p_{2}}{2 \pi \alpha_{E M} v} \\
g_{\sigma g g} & =\frac{i \alpha_{S} c_{g g} \epsilon p_{1}^{\mu_{2}} p_{2}^{\mu_{1}} \delta_{a_{1}, a_{2}}}{2 \pi v}-\frac{i \alpha_{S} c_{g g} \epsilon \delta_{a_{1}, a_{2}} g_{\mu_{1} \mu_{2}} p_{1} \cdot p_{2}}{2 \pi v} \\
g_{\sigma W W} & =\frac{2 i \epsilon m_{W}^{2} g_{\mu_{1} \mu_{2}}}{v} \\
g_{\sigma Z Z} & =\frac{2 i \epsilon m_{Z}^{2} g_{\mu_{1} \mu_{2}}}{v} \\
g_{\sigma f f} & =\frac{i \epsilon m_{f} \delta_{m_{1}, m_{2}} \delta_{s_{1}, s_{2}}}{v}
\end{aligned}
$$

where $\mathrm{f}$ is a fermion. The Lorentz indices $\mu_{i}$ run from 0 to 3 , and the gauge indices $a$ run from 1 to 8 The self-coupling of the dilaton is

$$
g_{\sigma \sigma \sigma}=-\frac{3 i \epsilon m_{\sigma}^{2}}{v}+\frac{i \epsilon \lambda m_{\sigma}^{2}}{v} .
$$

\section{A.2 Georgi-Machacek Model with Scalar DM}

\section{Triple scalar couplings}

The Feynman rules for couplings to $S$ are given by $-i g_{S S h_{i}}$ with all particles incoming and the couplings defined as follows:

$$
\begin{aligned}
& g_{S S h}=-4\left(\lambda_{a} s_{\alpha} v_{\phi}+\sqrt{3} \lambda_{b} c_{\alpha} v_{\chi}\right) \\
& g_{S S H}=-4\left(\lambda_{a} c_{\alpha} v_{\phi}+\sqrt{3} \lambda_{b} s_{\alpha} v_{\chi}\right) .
\end{aligned}
$$

Here the shorthand notation $s_{\alpha} \equiv \sin \alpha$ and $c_{\alpha} \equiv \cos \alpha$ is used. 


\section{Four scalar couplings}

The Feynman rules for couplings to $S$ are given by $-i g_{S S s_{1} s_{2}}$ with all particles incoming and the couplings defined as follows:

$$
\begin{aligned}
g_{S S h h} & =-4\left(\lambda_{a} c_{\alpha}^{2}+\lambda_{b} s_{\alpha}^{2}\right) \\
g_{S S H H} & =-4\left(\lambda_{a} s_{\alpha}^{2}+\lambda_{b} c_{\alpha}^{2}\right) \\
g_{S S h H} & =-4 s_{\alpha} c_{\alpha}\left(\lambda_{a}-\lambda_{b}\right) \\
g_{S S G^{0} G^{0}} & =g_{S S G^{+} G^{+*}}=-4\left(\lambda_{a} c_{H}^{2}+\lambda_{b} s_{H}^{2}\right) \\
g_{S S H_{3}^{0} H_{3}^{0}} & =g_{S S H_{3}^{+} H_{3}^{+*}}=-4\left(\lambda_{a} s_{H}^{2}+\lambda_{b} c_{H}^{2}\right) \\
g_{S S G^{0} H_{3}^{0}} & =g_{S S G^{+} H_{3}^{+*}}=g_{S S H_{3}^{+} G^{+*}}=-4 s_{H} c_{H}\left(\lambda_{b}-\lambda_{a}\right) \\
g_{S S H_{5}^{0} H_{5}^{0}} & =g_{S S H_{5}^{+} H_{5}^{+*}}=g_{S S H_{5}^{++} H_{5}^{++*}}=-4 \lambda_{b} .
\end{aligned}
$$

Here the shorthand notation $s_{H} \equiv \sin \theta_{H}$ and $c_{H} \equiv \cos \theta_{H}$ is used. 